\title{
Nature and evolution of the dominant carbonaceous matter in interplanetary dust particles: effects of irradiation and identification with a type of amorphous carbon
}

\author{
G. M. Muñoz Caro ${ }^{1,2}$, G. Matrajt ${ }^{1,3}$, E. Dartois ${ }^{1}$, M. Nuevo ${ }^{1,4}$, L. d'Hendecourt ${ }^{1}$, D. Deboffle ${ }^{1}$, G. Montagnac ${ }^{5}$, \\ N. Chauvin ${ }^{6}$, C. Boukari ${ }^{6}$, and D. Le Du ${ }^{6}$ \\ 1 Institut d'Astrophysique Spatiale, UMR 8617, Bât. 121, Université Paris XI, 91405 Orsay, France \\ 2 Centro de Astrobiología, INTA-CSIC, Carretera de Ajalvir, km. 4, Torrejón de Ardoz, 28850 Madrid, Spain \\ e-mail: munozcg@inta.es \\ 3 Department of Astronomy, Box 351580, University of Washington, Seattle, WA 98195, USA \\ 4 Institute of Astronomy, National Central University, No. 300, Jhongda Rd, Jhongli City, Taoyuan County 32049, Taiwan \\ 5 Laboratoire de Sciences de la Terre, ENS Lyon, 46 allée d'Italie, 69007 Lyon, France \\ ${ }^{6}$ CSNSM, IN2P3/CNRS, Campus Paris XI, 91405 Orsay, France
}

Received 17 December 2004 / Accepted 28 June 2006

ABSTRACT

\begin{abstract}
Aims. Interplanetary dust particle (IDP) matter probably evolved under irradiation in the interstellar medium (ISM) and the solar nebula. Currently IDPs are exposed to irradiation in the Solar System. Here the effects of UV and proton processing on IDP matter are studied experimentally. The structure and chemical composition of the bulk of carbon matter in IDPs is characterized.

Methods. Several IDPs were further irradiated in the laboratory using ultraviolet (UV) photons and protons in order to study the effects of such processing. By means of infrared and Raman spectroscopy, IDPs were also compared to different materials that serve as analogs of carbon grains in the dense and diffuse ISM.

Results. The carbonaceous fraction of IDPs is dehydrogenated by exposure to hard UV photons or $1 \mathrm{MeV}$ protons. On the other hand, proton irradiation at lower energies $(20 \mathrm{keV})$ leads to an efficient hydrogenation of the carbonaceous IDP matter. The dominant type of carbon in IDPs, observed with Raman and infrared spectroscopy, is found to be either a form of amorphous carbon ( $a$-C) or hydrogenated amorphous carbon $(a-\mathrm{C}: \mathrm{H})$, depending on the IDP, consisting of aromatic units with an average domain size of $1.35 \mathrm{~nm}$ (5-6 rings in diameter), linked by aliphatic chains.

Conclusions. The D- and ${ }^{15} \mathrm{~N}$-enrichments associated to an aliphatic component in some IDPs are probably the result of chemical reactions at cold temperatures. It is proposed that the amorphous carbon in IDPs was formed by energetic processing (UV photons and cosmic rays) of icy grains, maybe during the dense cloud stage, and more likely on the surface of the disk during the T Tauri phase of our Sun. This would explain the isotopic anomalies and morphology of IDPs. Partial annealing, $300-400{ }^{\circ} \mathrm{C}$, is required to convert an organic residue from ice photoprocessing into the amorphous carbon with low heteroatom content found in IDPs. Such annealing might have occurred as the particles approached the Sun and/or during atmospheric entry heating.
\end{abstract}

Key words. infrared: ISM - infrared: solar system - methods: laboratory - molecular processes

\section{Introduction}

Interplanetary dust particles (IDPs), with sizes ranging from a few microns to tens of microns, are collected by NASA aircrafts from the Earth's stratosphere. These particles mainly consist of silicate grains coated by carbonaceous material (Brownlee 1978). IDPs have an average carbon content of 10-12\% (Schramm et al. 1989). They often show an aliphatic component, responsible for the infrared $3.4 \mu \mathrm{m}$ band $\left(2800-3000 \mathrm{~cm}^{-1}\right.$, $\mathrm{CH}$ str.) and sometimes for carbonyl $(\mathrm{C}=\mathrm{O})$ groups, with str. absorption around $1700 \mathrm{~cm}^{-1}$ in the infrared (Flynn et al. 2003, 2004; Matrajt et al. 2005). Keller et al. (2004) propose that the aliphatic hydrocarbons are likely to occur as branches or side bands on more complex macromolecular material. Previous attempts to characterize the nature of the carbonaceous fraction in IDPs by electron energy loss spectroscopy (Keller et al. 1994) and carbon-X-ray absorption near edge structure measurements (Bajt et al. 1996), indicate that graphitic or amorphous carbon is the dominant type of carbon present in some of the IDPs.
The amorphous carbon characterization, however, has been put in doubt by Flynn et al. (2004), suggesting that approximately one-half of the total of $12 \mathrm{wt} \%$ carbon in typical IDPs is present as organic carbon.

Chondritic IDPs are generally classified by their silicate content (Sandford \& Walker 1985; Bradley et al. 1992): anhydrous IDPs contain pyroxenes and olivines and hydrated IDPs contain layer silicates. The formation mechanism of the carbon fraction of IDPs and the environment in which this material formed are currently unknown. The fact that both anhydrous and hydrated IDPs contain organic matter of similar types and abundances seems to indicate that the organic content in IDPs is not produced by aqueous alteration of carbonaceous material on asteroids, and suggests either a solar nebula or an interstellar origin (Flynn et al. 2003). Furthermore, $\mathrm{H}$ and $\mathrm{N}$ isotopic anomalies in the carbonaceous component of IDPs support that such material was formed by low-temperature reactions, although mass fractionation during dynamic pyrometamorphism deserves consideration (Rietmeijer 1998). This led some authors to the 
conclusion that the carbon IDP fraction originated in an interstellar molecular cloud environment (e.g. Keller et al. 2004).

This article reports on the exposure of several IDPs to UV and proton irradiation in order to study the effects of such processing on the carbonaceous and silicate fractions of IDPs. This provides constraints on the UV and proton fluences to which IDPs were exposed in interplanetary or interstellar environments and sheds light on their history of energetic processing. The evolution of the carbonaceous IDP matter in space is most likely connected to the interplay between UV and high energy cosmic rays, leading to dehydrogenation, and low-energy protons (a few $\mathrm{keV}$ ) or atomic hydrogen leading to rehydrogenation. In addition, the annealing experienced by IDPs during atmospheric entry should be considered (Rietmeijer 1998). To test this, we annealed relevant laboratory analogs at different temperatures.

The infrared and Raman spectra of IDPs were compared to those of laboratory analogs of carbon grains residing in diffuse and dense cloud environments. We provide a characterization of the dominant carbonaceous IDP matter that is consistent with the comparison to laboratory analogs. Based on these laboratory results, we elucidate a possible scenario for the formation and evolution of the bulk of the carbon component in IDPs.

The layout of this paper is as follows: the experimental protocols and the micro-FTIR and Raman analysis are described in Sect. 2. The experimental results are presented and evaluated in Sect. 3. The final discussion and astrophysical implications are given in Sect. 4.

\section{Experimental}

The IDPs used for this project were provided by the NASA-JSC curation facility. They varied in size from 10 to $30 \mu \mathrm{m}$. Each IDP was stored between two glass plates. IDPs were examined and handled under an optical microscope in a laminarflow 10000 -class clean room. The particles were rinsed 5 times with high purity hexane (Aldrich) to remove the silicon oil used in the collectors to decelerate the particles. Subsequently, the particles were transfered to a KRS-5 (50\% Thalium Bromide, $50 \%$ Thalium Iodide) infrared-transparent window, resistant to $\mathrm{HF}$, with a brush hair mounted in a micromanipulator. The particles were squeezed onto the KRS-5 window using a previouslycleaned glass plate. Some of the particles were treated with HF to etch and remove the silicates (Matrajt et al. 2005).

\subsection{FTIR analysis}

Fourier-transform infrared spectroscopy was performed with the use of a Nicolet Magna-IR 560 ESP spectrometer coupled to a Nicolet Nicplan infrared microscope, in line with a synchrotron radiation source located on line SU5 at the Laboratoire pour l'Utilisation du Rayonnement Electromagnétique (LURE) at the University of Paris-Sud, Orsay, France. The spectrometer is equipped with a $\mathrm{KBr}$ beamsplitter and a nitrogen-cooled Mercury-Cadmium-Tellure (MCT) detector working in the 4000 to $650 \mathrm{~cm}^{-1}(2.5$ to $15 \mu \mathrm{m})$ spectral range. The microscope uses a Schwarzchild-Cassegrain objective (32x) and a condenser (10x) in the transmission mode. We used the standard internal light source of the instrument, a conventional black body-like source (Globar) that is very stable and allows spectroscopic analysis of samples larger than about $6 \mu \mathrm{m}$ (Raynal et al. 2000b). For the spectra of IDPs, it is important to enhance the signal-to-noise ratio and minimize background fluctuations caused by purge variations. The spectra presented here are the average of 25 acquisitions of 256 scans each at resolution $2 \mathrm{~cm}^{-1}$.
The infrared spectra of IDPs were usually corrected for lightscattering effects using a spline baseline through regions where there are no absorption features. Integration of the absorbance area of the features was performed using an in-house IDL code. The column densities for aliphatics ( $N$ [3.4], in aliphatic $\mathrm{C}$ atoms $\mathrm{cm}^{-2}$ ), are also given. These column densities were determined using the formula

$N=\int_{\text {band }} \frac{\tau_{\nu} \mathrm{d} v}{A}$

where $N$ is the column density in $\mathrm{cm}^{-2}, \tau$ the optical depth of the band, $\mathrm{d} v$ the wavenumber differential in $\mathrm{cm}^{-1}$, and $A$ the band strength in $\mathrm{cm} \mathrm{molec}^{-1}$.

We calculated the column density of aliphatic $\mathrm{C}$ atoms as $N[3.4]=N\left(\mathrm{CH}_{2}\right)+N\left(\mathrm{CH}_{3}\right)$, where the absorbance areas of the peaks at 2958 and $2920 \mathrm{~cm}^{-1}$ (asymmetric $\mathrm{CH}_{3}$ and $\mathrm{CH}_{2}$ str. modes) are calculated using a Gaussian fit of the data. The adopted band strengths, $A\left(\mathrm{CH}_{3}\right)=1.25 \times 10^{-17} \mathrm{~cm}(\mathrm{C} \text { atom })^{-1}$ and $A\left(\mathrm{CH}_{2}\right)=8.4 \times 10^{-18} \mathrm{~cm}(\mathrm{C} \text { atom })^{-1}$, are those given in Dartois et al. (2004) for hexane ice.

For the evaluation of the column density of the silicate band around $\sim 1000 \mathrm{~cm}^{-1}(N[10]), A[10]=2.0 \times 10^{-16} \mathrm{~cm}(\mathrm{Si} \text { atom })^{-1}$ was used (Matrajt et al. 2005 and ref. therein).

\subsection{Micro-Raman analysis}

The samples were analyzed in Lyon, using a Raman spectrometer of the Laboratoire de Sciences de la Terre (Labram HR800 vis Jobin Yvon, coupled to a Spectra Physics Argon ${ }^{+}$laser tuned for these experiments in the green part $(514.5 \mathrm{~nm})$ of the visible spectrum). The laser beam is focussed through microscope objectives $(\times 50)$ down to a $2 \mu \mathrm{m}$ spot on samples, and the backscattered light is collected through the same objective. For Raman spectra, a 1800-lines grating was used in a wavelength range centered around the $\mathrm{D}$ and $\mathrm{G}$ carbonaceous lines $\left(\sim 1600 \mathrm{~cm}^{-1}\right)$, giving a resolution of $\lambda / \Delta(\lambda) \approx 600$. Wavelength calibration was checked against a silicon wafer sample, and it agreed within better than $1 \mathrm{~cm}^{-1}$ for all the experiments. Care was taken to irradiate the samples with low fluxes, in order not to modify their structures, by interposing film densities (typically densities of 2 to 4 ). The laser power experienced by the samples was well below $1 \mathrm{~mW}$.

\section{Experimental results}

In this section, the results obtained from experiments dealing with UV- and $\mathrm{H}^{+}$-irradiation of IDPs are reported and discussed. Raman spectroscopy is used to characterize the carbon bulk in IDPs. By means of FTIR and Raman spectroscopy, the carbon fraction in IDPs is compared to laboratory analogs of i) molecular cloud organics and ii) diffuse medium carbonaceous grains. The results of these comparisons provide further evidence for the characterization of the carbon bulk in IDPs.

\subsection{UV-irradiation of IDPS}

We submitted several IDPs to UV under vacuum $(P \approx 1 \times$ $10^{-7}$ mbar) to see the evolution of the organic features as a function of irradiation, using a microwave-stimulated hydrogenflow discharge lamp. The value of the UV flux was assumed to be the one measured by Weber \& Greenberg (1985) for the same lamp model (output $\approx 1.5 \times 10^{15}$ photons $^{-1}$ and $E_{\text {photon }}=7.3-10.5 \mathrm{eV}$, see Muñoz Caro \& Schutte 2003 for lamp 

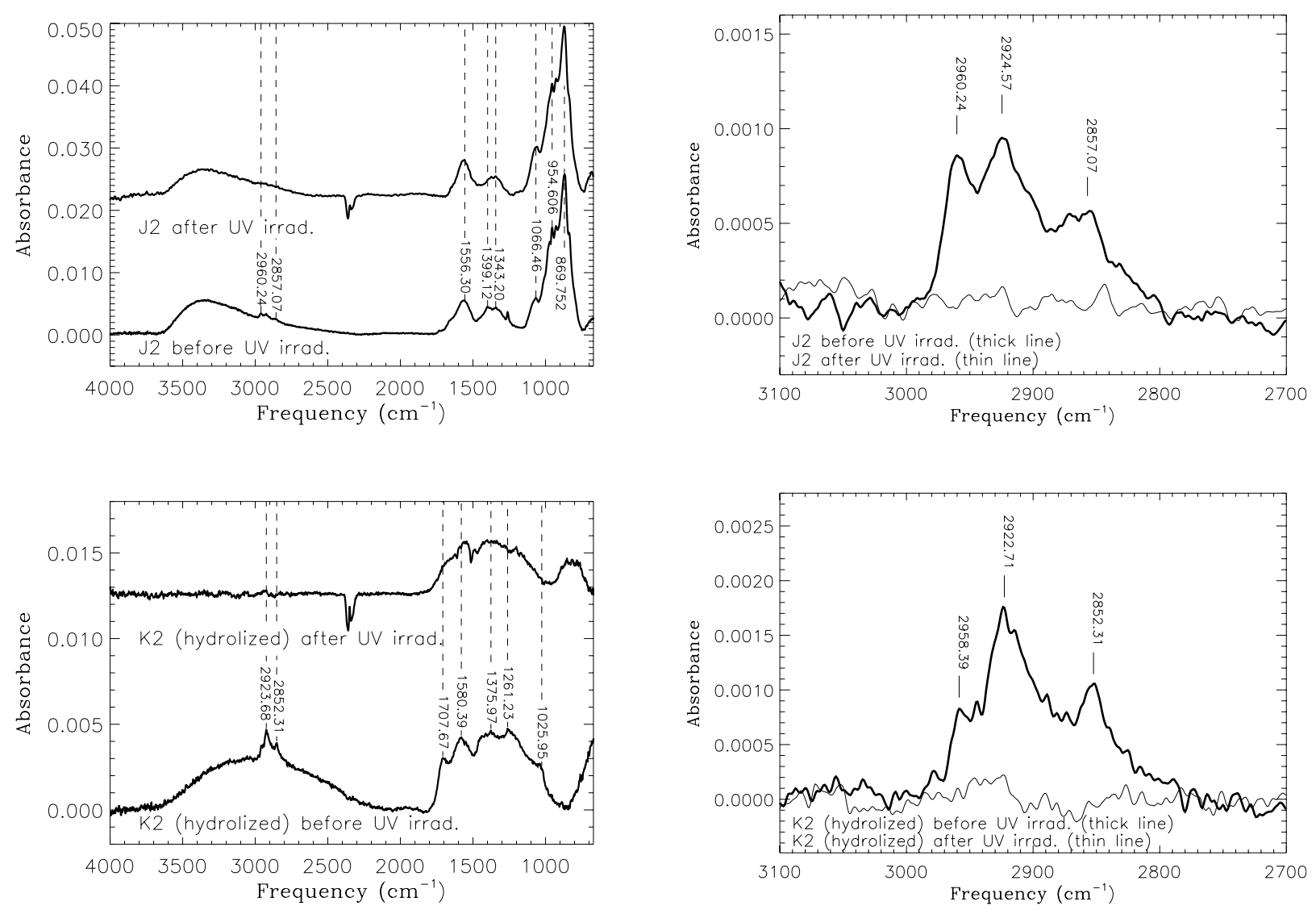

Fig. 1. Left: infrared spectrum of IDPs before and after UV-irradiation. Right: blow up of the $3.4 \mu \mathrm{m}$ region corresponding to spectra in the left panels after subtraction of a local spline baseline. Particle K2 (bottom) was treated previously with HF to remove the silicates.

Table 1. Experimental parameters corresponding to IDPs used in this work and before and after energetic processing. See text in Sects. 3.1 and 3.2 for explanation.

\begin{tabular}{|c|c|c|c|c|c|c|}
\hline IDP & fluence & area[3.4] & $\begin{array}{c}N[3.4] \\
\left(\text { aliph. } \mathrm{C} \mathrm{cm}^{-2}\right)\end{array}$ & $\frac{\overline{N\left(\mathrm{CH}_{2}\right)}}{N\left(\mathrm{CH}_{3}\right)}$ & area[10] & $\begin{array}{c}N[10] \\
\left(\mathrm{Si} \mathrm{cm}^{-2}\right)\end{array}$ \\
\hline J2 (cluster, $\mathrm{Px}+\mathrm{Oli}$ ) & & 0.063 & $1.4 \times 10^{16}$ & 2.9 & 3.39 & $3.9 \times 10^{16}$ \\
\hline $\mathrm{J} 2$ (cluster, $\mathrm{Px}+\mathrm{Oli}$ ) & $3.2 \times 10^{19}$ UV phot. $\mathrm{cm}^{-2}$ & $\leq 0.01$ & $\leq 2.2 \times 10^{15}$ & - & - & - \\
\hline K2 (cluster, LLS+Oli), HF hyd. & & 0.17 & $1.9 \times 10^{16}$ & 5.5 & 0.90 & $1.1 \times 10^{16}$ \\
\hline K2 (cluster, LLS+Oli), HF hyd. & $7.2 \times 10^{19}$ UV phot. $\mathrm{cm}^{-2}$ & $\leq 0.02$ & $\leq 2.2 \times 10^{15}$ & - & - & - \\
\hline R9 (cluster, Px+Oli) & & 0.14 & $2.0 \times 10^{16}$ & 5.5 & 7.24 & $8.41 \times 10^{16}$ \\
\hline R9 (cluster, $\mathrm{Px}+\mathrm{Oli}$ ) & $10^{16}\left(20 \mathrm{keV} \mathrm{H}^{+}\right) \mathrm{cm}^{-2}$ & 0.30 & $4.3 \times 10^{16}$ & 2.9 & - & - \\
\hline R9 (cluster, Px+Oli) & $10^{16}\left(20 \mathrm{keV}+1 \mathrm{MeV} \mathrm{H}^{+}\right) \mathrm{cm}^{-2}$ & $\leq 0.05$ & $\leq 6.3 \times 10^{15}$ & - & - & - \\
\hline $\mathrm{N}$ (chondritic, LLS+Px+Oli), HF hyd. & & 0.20 & $2.6 \times 10^{16}$ & 2.8 & - & - \\
\hline $\mathrm{N}$ (chondritic, LLS+Px+Oli), HF hyd. & $10^{16} 1 \mathrm{MeV} \mathrm{H}^{+} \mathrm{cm}^{-2}$ & $\leq 0.03$ & $\leq 3.9 \times 10^{15}$ & - & - & - \\
\hline
\end{tabular}

spectrum). The flux at the sample position is then estimated to be $\approx 5 \times 10^{14}$ photons $\mathrm{cm}^{-2} \mathrm{~s}^{-1}$.

Figure 1, left column, shows the infrared spectra of the IDPs before (bottom spectrum) and after (top spectrum) they were exposed to UV-irradiation. Panels in the right column show a zoom of the $3.4 \mu \mathrm{m}$ region after subtraction of a local spline baseline. Table 1 provides the experimental parameters of the irradiation experiments corresponding to IDPs L2054-J2, L2036-R9 (not hydrolized), L2021-K2, and W7116B-N (hydrolized with HF), respectively abbreviated as $\mathrm{J} 2, \mathrm{R} 9, \mathrm{~K} 2$, and $\mathrm{N}$. The first column gives the name of the particle, the type (chondritic or cluster), and the silicate content $(\mathrm{Px}=$ pyroxene, $\mathrm{Oli}=$ olivine, $\mathrm{LLS}=$ layer lattice silicate). The second column gives the fluence (for $\mathrm{UV}$-irradiation it corresponds to the product $\phi_{\mathrm{UV}} t$ where $\phi_{\mathrm{UV}} \approx$ $5 \times 10^{14}$ photons $\mathrm{cm}^{-2} \mathrm{~s}^{-1}$ is the UV-flux at sample position and $t$ is the irradiation time, $18 \mathrm{hr}$. for $\mathrm{J} 2$ and $40 \mathrm{hr}$. for $\mathrm{K} 2$ ). The third and fourth columns are, respectively the absorbance area, area[3.4], and column density, $N$ [3.4], of the $3.4 \mu \mathrm{m}$ feature in the region $2800-3000 \mathrm{~cm}^{-1}$, corresponding to the $\mathrm{CH}$ str. of aliphatic species. The fifth column gives the ratio between the column densities of the $\mathrm{CH}_{2}$ and $\mathrm{CH}_{3}$ groups, $N\left(\mathrm{CH}_{2}\right) / N\left(\mathrm{CH}_{3}\right)$. The last two columns provide the absorbance area, area[10] and column density, $N$ [10], of the $10 \mu \mathrm{m}$ feature (around $1000 \mathrm{~cm}^{-1}$ ) of the silicates. The absorbance values were calculated after a spline baseline correction.

The experimental results obtained from the UV-irradiation of IDPs (see Fig. 1 and Table 1) show that the $3.4 \mu \mathrm{m}$ feature is reduced by at least $85 \%$ for particles $\mathrm{J} 2$ and $\mathrm{K} 2$ after irradiation. For particle $\mathrm{K} 2$, the band at $1707 \mathrm{~cm}^{-1}$ assigned to a carbonyl $(\mathrm{C}=\mathrm{O})$ stretch also seems to disappear. 
Table 2. Values of the nuclear, $S_{\mathrm{n}}$, and electron, $S_{\mathrm{e}}$, stopping powers and penetration ranges, $R$, provided by TRIM for San Carlos olivine, silicone, and a hydrocarbon material with $\mathrm{C}: \mathrm{H}=1: 1$ as target materials, using $\mathrm{H}^{+}$ions with kinetic energy $E$.

\begin{tabular}{cccccc}
\hline \hline Material & ion & $E[\mathrm{keV}]$ & $S_{\mathrm{n}}\left[\mathrm{eV} / \AA \AA^{\mathrm{A}}\right]$ & $S_{\mathrm{e}}\left[\mathrm{eV} / \AA \AA^{\mathrm{A}}\right]$ & $R[\mu \mathrm{m}]$ \\
\hline San Carlos olivine & $\mathrm{H}^{+}$ & 20 & 0.07 & 9.6 & 0.24 \\
San Carlos olivine & $\mathrm{H}^{+}$ & 1000 & 0.003 & 4.6 & 14.5 \\
San Carlos olivine & $\mathrm{H}^{+}$ & 1500 & 0.002 & 3.6 & 26.7 \\
Silicone & $\mathrm{H}^{+}$ & 20 & 0.06 & 10.1 & 0.23 \\
Silicone & $\mathrm{H}^{+}$ & 1000 & 0.003 & 4.1 & 16.33 \\
Hydrocarbon C:H $=1: 1$ & $\mathrm{H}^{+}$ & 20 & 0.055 & 8.1 & 0.33 \\
Hydrocarbon C:H $=1: 1$ & $\mathrm{H}^{+}$ & 1000 & 0.002 & 2.9 & 21.5 \\
\hline
\end{tabular}

In experiments involving UV-photoprocessing, it is crucial to check whether the irradiated sample is optically thin, given the short penetration length of UV-photons. Experiments on photoprocessing of a hexane ice layer with an absorbance value of the $3.4 \mu \mathrm{m}$ feature comparable to that of the IDPs studied here, led to $95 \%$ destruction of the band after $3 \mathrm{hr}$. of irradiation (Muñoz Caro et al. 2001). As a result, new features were observed around 1650 and $2100 \mathrm{~cm}^{-1}$, respectively, assigned to doubly and triply unsaturated C-C bonds that provide direct evidence of the dehydrogenation of the samples. The formation of additional bands due to unsaturated species was not observed in the irradiated IDPs, maybe due to the low intrinsic strengths of these features. Also the $3.4 \mu \mathrm{m}$ feature of more complex carbonaceous materials, like a type of hydrogenated carbon grain (called ACH2, see Sect. 3.4.3) is very efficiently reduced upon photolysis (Mennella et al. 2001). As a first approximation, assuming a constant destruction cross section, the photodestruction of the $\mathrm{CH}$ bonds is expected to lead to an exponential decay of the integrated intensity of the $3.4 \mu \mathrm{m}$ feature, $\tau$, expressed as

$$
\tau=\tau_{0} \cdot \exp -\left(\phi_{\mathrm{UV}} \cdot \sigma_{\mathrm{des}, \mathrm{UV}} \cdot t\right)
$$

where $\tau_{0}$ is the initial integrated intensity of the feature, $\phi_{\mathrm{UV}}$ the flux at the sample position in UV photon $\mathrm{cm}^{-2} \mathrm{~s}^{-1}, \sigma_{\text {des,UV }}$ the destruction cross section of $\mathrm{CH}$ bonds per UV photon, and $t$ the irradiation time in seconds.

Using the values for the UV fluence and $N$ [3.4] given in Table 1 , and assuming $\sigma_{\text {des, UV }}=1.0 \times 10^{-19} \mathrm{~cm}^{2}$ UV-photon ${ }^{-1}$ obtained for ACH2 (Mennella et al. 2001), it is found that $95 \%$ of the $3.4 \mu \mathrm{m}$ feature would be destroyed for particle $\mathrm{J} 2$ and $99 \%$ for $\mathrm{K} 2$ if the carbon phase in the IDPs is similar to $\mathrm{ACH} 2$, in agreement with the minimum of $85 \%$ destruction we reported above. However, the absorption of the silicate phase in these particles should be taken into account. The absorption of the remaining silicates in particle $\mathrm{K} 2$, treated with $\mathrm{HF}$, is $N[10]=$ $1.1 \times 10^{16} \mathrm{Si}$ atoms $\mathrm{cm}^{-2}$ (Matrajt et al. 2005), which roughly corresponds to $\tau_{\mathrm{UV}}[10] \sim 0.32$, using a UV cross-section of $2.9 \times 10^{-17} \mathrm{~cm}^{2}$ (Si atom) $)^{-1}$ (Witt et al. 1998). For particle J2, $N[10]=3.9 \times 10^{16} \mathrm{Si}$ atoms $\mathrm{cm}^{-2}$, giving $\tau_{\mathrm{UV}}[10] \sim 1.1$, which is optically thick. Therefore, the remaining silicate abundance in the hydrolized IDP K2 is expected to absorb only a fraction of the UV photons and should not prevent the aliphatic component in IDPs from photolysis. This is even more valid if we consider that the silicate phase in IDPs is not on top of the organics; instead, the silicate grains are generally coated by the organic phases (e.g. Wopenka 1988). It is thus expected that even the particles that were not hydrolized, therefore preserving the original silicates like J2 with $\tau_{\mathrm{UV}}[10]>1$ (thick in the UV region), will still have a significant fraction of their organics exposed to the UV photons. This explains the $\geq 85 \% \mathrm{CH}$ bond destruction reported above for IDP J2. In conclusion, as occurs to simple aliphatic chains and ACH2 (Muñoz Caro et al. 2001;
Mennella et al. 2001), UV irradiation of IDPs leads to the destruction of most of their aliphatic $\mathrm{CH}$ bonds.

\section{2. $\mathrm{H}^{+}$-irradiation of IDPs}

Two IDPs ( $\mathrm{R} 9$ and $\mathrm{N}$ ) were irradiated with an $\mathrm{H}^{+}$ion beam at two different energies, $20 \mathrm{keV}$ and $1 \mathrm{MeV}$, under vacuum $\left(10^{-7} \mathrm{mbar}\right)$ at room temperature. The fluence was $10^{16}$ atoms $\mathrm{cm}^{-2}$, and the beam intensity was maintained below $5 \mu \mathrm{A}$ at $20 \mathrm{keV}$ and below $1 \mu \mathrm{A}$ at $1 \mathrm{MeV}$. These experiments were performed at CSNSM, located in Orsay. Low energy irradiation $(20 \mathrm{keV})$ was achieved with the isotope separator SIDONIE (Chauvin et al. 2004) and high energy irradiation $(1 \mathrm{MeV})$ with the use of the electrostatic tandem accelerator ARAMIS (Bernas et al. 1992). The IDPs were analyzed before and after irradiation by means of micro-FTIR spectroscopy.

For different target materials, we estimated the values of the nuclear, $S_{\mathrm{n}}$, and electron, $S_{\mathrm{e}}$, stopping powers and penetration ranges, $R$, for $\mathrm{H}^{+}$ions at the relevant kinetic energies, $E$, using the TRIM Montecarlo simulation program (Table 2). San Carlos olivine was selected as representative of the silicate phase in IDP R9. The choice of silicate as the target material does not affect the calculated parameter values considerably (silicone, for instance, gives similar values to olivine, see Table 2). A hydrocarbon with $\mathrm{C}: \mathrm{H}=1: 1$ was adopted as a crude representative of the carbon fraction in IDPs.

Figure 2 illustrates the results obtained by irradiation of IDPs R9 and N, see caption for description. The experimental parameters corresponding to $\mathrm{H}^{+}$-irradiation of IDPs $\mathrm{R} 9$ and $\mathrm{N}$ are given in Table 1 (see Sect. 3.1 for table description). The bottom left panel in Fig. 2 shows that the $3.4 \mu \mathrm{m}$ feature has increased considerably as a result of $20 \mathrm{keV}-\mathrm{H}^{+}$implantation on the carbonaceous phase of IDP R9. The $N$ [3.4] values for R9 in Table 1 show that the number of $\mathrm{C}$ atoms forming $\mathrm{CH}$ bonds increased by a factor 2.15, which indicates that the IDP was efficiently hydrogenated. In fact, if we consider that $\mathrm{CH}_{2}$ and $\mathrm{CH}_{3}$ bonds respectively need 2 and $3 \mathrm{H}^{+}$ions to form, the $\mathrm{H}^{+}$fluence required is at least $5.4 \times 10^{16} \mathrm{H}^{+} \mathrm{cm}^{-2}$, much higher than the $1 \times 10^{16} \mathrm{H}^{+} \mathrm{cm}^{-2}$ value that was intended. The possibility of hydrocarbon contamination is very remote, so the likely explanation is that the reported value of the fluence, $1 \times 10^{16} \mathrm{H}^{+} \mathrm{cm}^{-2}$, is not reliable. As a result of $\mathrm{H}^{+}$irradiation, the aliphatic $\mathrm{CH}_{3}$ sym. str. absorption, rarely observed in IDPs, becomes recognizable (at $2872 \mathrm{~cm}^{-1}$, bottom left panel in Fig. 2). Another effect of the irradiation is that the ratio $N\left(\mathrm{CH}_{2}\right) / N\left(\mathrm{CH}_{3}\right)$ decreased from 5.5 to 2.9 , indicating that the aliphatic chains became more branched. Before $20 \mathrm{keV}-\mathrm{H}^{+}$irradiation, a weak absorption around $2898 \mathrm{~cm}^{-1}$ seemed to be present, that could be due to olefinic $\mathrm{CH}$ groups. This band is not clearly observed after irradiation, which is compatible with the strong hydrogenation that took place. 

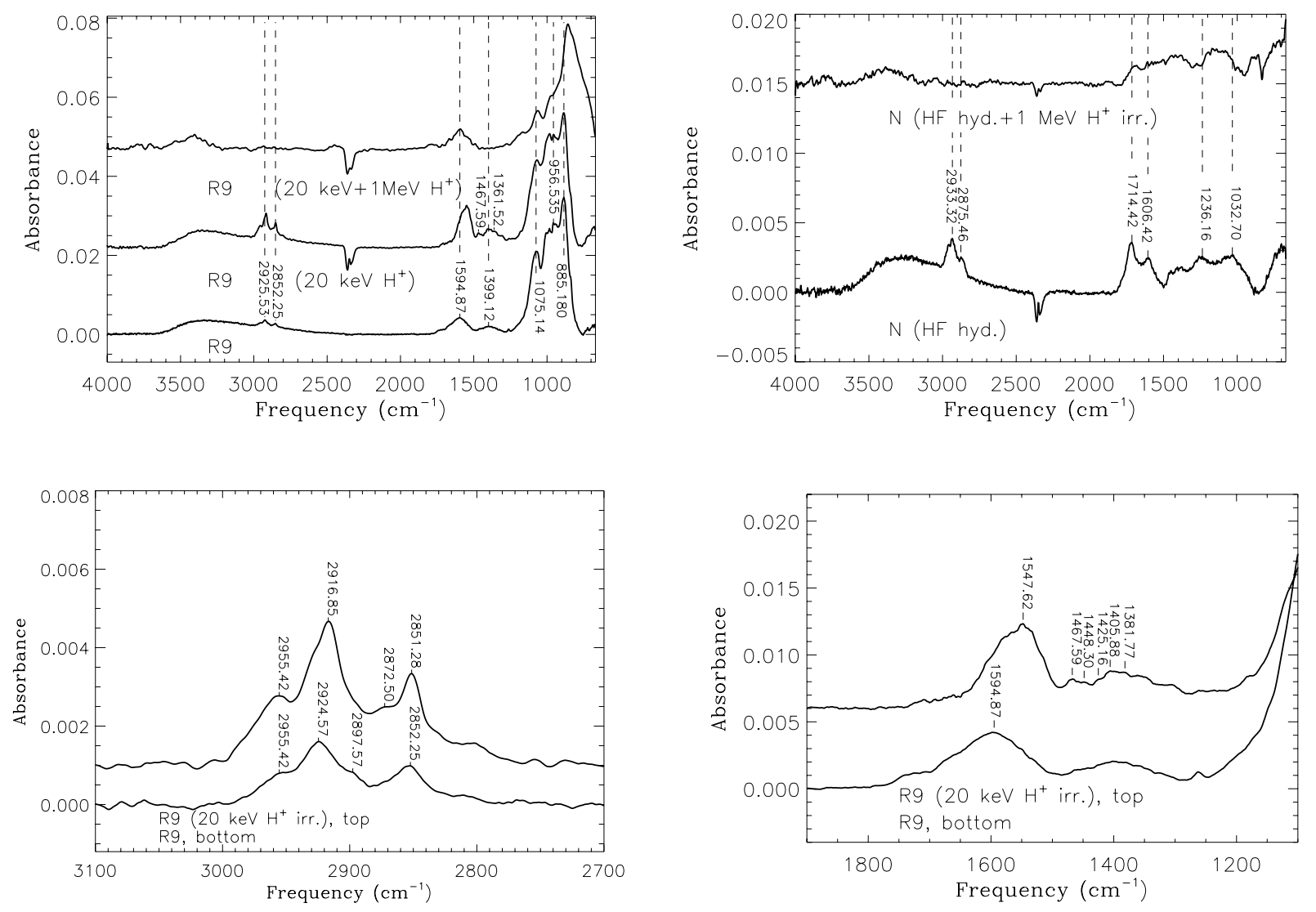

Fig. 2. Top left: infrared spectrum of IDP R9 before irradiation (low trace), after $20 \mathrm{keV} \mathrm{H}^{+}$irradiation (middle trace), and after $20 \mathrm{keV}+1 \mathrm{MeV} \mathrm{H}{ }^{+}$ irradiation. Top right: infrared spectrum of HF-hydrolized IDP $\mathrm{N}$ before and after $1 \mathrm{MeV} \mathrm{\textrm {H } ^ { + }}$ irradiation. Bottom left: blow-up of the $3.4 \mu \mathrm{m}$ feature corresponding to bottom and middle spectra in the top left panel after subtraction of a local spline baseline. Bottom right: blow-up of the 1900-1100 $\mathrm{cm}^{-1}$ region, showing the absorptions due to the $\mathrm{CH}$ bending modes, corresponding to bottom and middle spectra in the top left panel.

Table 3. Vibrational assignment of $\mathrm{CH}$ bending modes corresponding to top spectrum in Fig. 2.

\begin{tabular}{cc}
\hline \hline Position $\left(\mathrm{cm}^{-1}\right)$ & Vibrational mode \\
\hline 1468 & aliph. $\mathrm{CH}_{3}$ asym. bending \\
1448 & aliph. $\mathrm{CH}_{2}$ bending \\
1425 & arom. $\mathrm{CH}$ bending \\
1406 & olef. $\left(\mathrm{CH}_{3}\right)_{3}$ sym. bending \\
1382 & aliph. $\mathrm{CH}_{3}$ sym. bending \\
\hline
\end{tabular}

The intrinsically weak bands assigned to the $\mathrm{CH}$ bending modes of IDP R9 are difficult to observe prior to irradiation (Fig. 2, bottom right panel, lower trace), despite the long integration time $\left(25 \times 256\right.$ scans with $2 \mathrm{~cm}^{-1}$, see Sect. 2.1). After $20 \mathrm{keV} \mathrm{H}^{+}$irradiation, the carbonaceous fraction of the IDP is efficiently hydrogenated and these bands become barely observable (Fig. 2, bottom right panel, upper trace), with poor signalto-noise ratios of $S / N \geq 3-5$, although they seem to be real absorptions, which is also appreciated in the middle trace of the top left panel in Fig. 2, due to the larger observable continuum. The possible assignments of some of these weak bands are given in Table 3, while others remain to be assigned. The carrier of the prominent band at $1548 \mathrm{~cm}^{-1}$ that appears after irradiation is unknown. The band at $1595 \mathrm{~cm}^{-1}$ in the spectrum before irradiation is likely due to the aromatic $\mathrm{C}=\mathrm{C}$ stretch. Hydrogenation of the carbon in the particle by the effect of $\mathrm{H}^{+}$irradiation could be responsible for the decrease in this band, as observed in the bottom right panel of Fig. 2. Indeed, the large increase in the number of $\mathrm{CH}$ bonds requires a decrease in the number of saturated $\mathrm{C}-\mathrm{C}$ bonds.

The estimated thickness of the crashed IDPs is at least $3 \mu \mathrm{m}$. From Table 2, the penetration range of $20 \mathrm{keV} \mathrm{H}^{+}$in hydrocarbons is about $0.3 \mu \mathrm{m}$, and thus at most $10 \%$ of the carbonaceous IDP phase was exposed to irradiation, although we suspect that the fraction of carbon exposed was larger, since the carbonaceous phase in IDPs is generally coating the silicates (Sect. 3.1). The exceedingly high hydrogenation of IDP R9 after $20 \mathrm{keV} \mathrm{H}^{+}$irradiation, described above, needs to be tested with new $\mathrm{H}^{+}$irradiation experiments of IDPs. Previous works report the hydrogenation of carbon materials by $\mathrm{keV} \mathrm{H}^{+}$irradiation. The formation of $\mathrm{CH}$ bonds on vapor-grown carbon fiber (VGCF), due to successive irradiations of 6,3 , and $1 \mathrm{keV}-\mathrm{H}^{+}$, has been observed by FTIR spectroscopy (Gotoh \& Kajiura 1999).

It follows from Table 1 that the irradiation with $1 \mathrm{MeV} \mathrm{H}^{+}$ of particle $\mathrm{N}$ leads to at least an $85 \%$ decrease of the $3.4 \mu \mathrm{m}$ feature. The carbonyl $(\mathrm{C}=\mathrm{O}$ str. $)$ band at $1714 \mathrm{~cm}^{-1}$ also seems to be reduced (Fig. 2, top right). The $1 \mathrm{MeV} \mathrm{H}^{+}$irradiation of particle R9, previously irradiated with $20 \mathrm{keV} \mathrm{H}^{+}$(see above), led to at least $90 \%$ decrease in the $3.4 \mu \mathrm{m}$ feature with respect to the same feature after $20 \mathrm{keV} \mathrm{H}^{+}$irradiation (Fig. 2, top left, and Table 1). Therefore, the $\mathrm{CH}$ bonds in the carbonaceous material of IDPs $\mathrm{N}$ and $\mathrm{R} 9$ were efficiently destroyed by $1 \mathrm{MeV}$ protons.

The estimated penetration range, $R$, of $1 \mathrm{MeV} \mathrm{H}^{+}$on a hydrocarbon with $\mathrm{C}: \mathrm{H}=1: 1$ is about $20 \mu \mathrm{m}$ and about $14 \mu \mathrm{m}$ for olivine (Table 2), so that a crashed IDP with a few $\mu \mathrm{m}$ thickness is fully exposed to irradiation. Similar to the case of 
Table 4. Overview of experimental parameters for $\mathrm{H}^{+}$irradiation of IDPs reported here and those reported in the literature for irradiation of interstellar silicate analogs.

\begin{tabular}{cccccc}
\hline \hline Work & Material & Sample thickness $[\mu \mathrm{m}]$ & $E[\mathrm{keV}]$ & Fluence[ion $\left.\mathrm{cm}^{-2}\right]$ & Amorphisation \\
\hline Brucato et al. (2004) & Forsterite & 0.038 & 30 & $7 \times 10^{15}$ & Negligible \\
Demyk et al. (2004) & Diopside & 0.140 & 10 & $10^{18}$ & Yes \\
Day (1977) & Olivine & 1 & 1500 & $7 \times 10^{17}$ & No \\
This work & R9 (IDP) & $>3$ & 20 & $>10^{16}$ & Negligible \\
This work & R9 (IDP) & $>3$ & 1000 & $10^{16}$ & Yes \\
\hline
\end{tabular}

UV-irradiation, Sect. 3.1, we can obtain an upper limit for the decrease of the $3.4 \mu \mathrm{m}$ feature as the result of $1 \mathrm{MeV} \mathrm{H}^{+}$irradiation, assuming a first order kinetic approach, using

$\tau=\tau_{0} \cdot \exp -\left(\phi_{1 \mathrm{MeV} \mathrm{H}^{+}} \cdot \sigma_{\mathrm{des}, 1 \mathrm{MeV} \mathrm{H}^{+}} \cdot t\right)$

where $\phi_{1 \mathrm{MeVH}^{+}} t=1 \times 10^{16}\left(1 \mathrm{MeV} \mathrm{H}^{+}\right) \mathrm{cm}^{-2}$ and assuming $\sigma_{\text {des, } 1 \mathrm{MeVH}^{+}}=9.4 \times 10^{-16} \mathrm{~cm}^{2}\left(1 \mathrm{MeV} \mathrm{H}^{+}\right)^{-1}$ (for ACARL_H, Mennella et al. 2003; see Sect. 3.4.3) as the destruction cross section of $\mathrm{CH}$ bonds per $1 \mathrm{MeV}$ proton, the expected maximum destruction for particle $\mathrm{N}$ would be above $99 \%$. The $85 \%$ and $90 \%$ lower limits for the destruction of $\mathrm{CH}$ bonds in IDPs $\mathrm{N}$ and R9 (see above) indicate that the destruction might be close to the $99 \%$ value of the first-order kinetic approach.

The shape and strength of the $10 \mu \mathrm{m}$ silicate band has drastically changed after irradiation, as a result of amorphisation of the silicates. Such a large effect is not observed in the case of $20 \mathrm{keV} \mathrm{H}^{+}$irradiation. Table 4 lists the parameters of other works dealing with $\mathrm{H}^{+}$irradiation of silicates. According to Brucato et al. (2004), amorphisation in forsterite depends on the nuclear elastic collisions between the impinging ions and the target ions, while they observed no correlation between the irradiation effects on the silicate structure and anaelastic electron collisions. As the ion energy, $E$, increases, the nuclear stopping power, $S_{\mathrm{n}}$, decreases, as can be seen in Table 2 . Indeed, experiments using low $E(10 \mathrm{keV}) \mathrm{H}^{+}$ions led to amorphisation of the silicate target (Table 4), while experiments with higher $E(30-1500 \mathrm{keV}) \mathrm{H}^{+}$ions led to negligible or no amorphisation. Our result agrees with the work of Brucato et al. (2004). As discussed above, the fluence corresponding to the $20 \mathrm{keV} \mathrm{H}^{+}$ion irradiation experiment was effectively much higher than $1 \times$ $10^{16} \mathrm{H}^{+} \mathrm{cm}^{-2}$. The estimated thickness of the crashed IDPs used for irradiation was at least $3 \mu \mathrm{m}$, about one order of magnitude larger than $R \sim 0.24 \mu \mathrm{m}$ for $20 \mathrm{keV} \mathrm{H}^{+}$(Table 2). This explains the poor silicate amorphisation for $20 \mathrm{keV} \mathrm{H}^{+}$ions, as observed by transmission FTIR. More surprising is the amorphisation induced by $1 \mathrm{MeV} \mathrm{H}^{+}$, since the thickness of the crashed IDP, not much larger than about $3 \mu \mathrm{m}$, is considerably lower than the calculated $R$, see Table 2. More experiments are needed on the irradiation of IDPs with high-energy $\mathrm{H}^{+}$ions to test the validity of this preliminary result.

\subsection{Micro-Raman spectroscopy of IDPs}

\subsubsection{Raman spectroscopy of carbonaceous materials}

Raman spectroscopy provides information on the chemical composition and structure of carbonaceous samples, making it a useful tool for probing the evolution of the material as an effect of irradiation and/or thermal annealing (Tuinstra \& Koening 1970). While microcrystalline graphite has a single line at $1582 \mathrm{~cm}^{-1}$ (graphitic or G line, assigned to zone-center phonons of $E_{2 g}$ symmetry), disordered graphite shows two lines around $1350 \mathrm{~cm}^{-1}$ (disorder or D line, assigned to $K$-point phonons of
$A_{1 g}$ symmetry) and $1580-1600 \mathrm{~cm}^{-1}$ (G line). The full width at half maximum $(F W H M)$ of the $\mathrm{D}$ band is related to the degree of graphitisation of the carbonaceous material: as the degree of order increases, the value of this parameter decreases. The position of the $\mathrm{G}$ band is related to the structural type of the carbon (nanocrystalline, polycrystalline, or amorphous) and, similar to the D band, its $F W H M$ increases as the degree of order decreases. The intensity ratio of the first order $\mathrm{D}$ and $\mathrm{G}$ lines, $I(\mathrm{D}) / I(\mathrm{G})$, is given by

$\frac{I(\mathrm{D})}{I(\mathrm{G})}=\frac{C(\lambda)}{L_{\mathrm{a}}}$

where $L_{\mathrm{a}}$ is the aromatic cluster diameter or in-plane correlation length and $C(515.5 \mathrm{~nm}) \sim 44 \AA$. This relation, however, is not valid for highly disordered carbon materials (Tuinstra \& Koenig 1970).

The effects of the amorphisation of graphite on the evolution of the Raman spectrum, following the sequence graphite $\rightarrow$ nanocrystalline graphite $\rightarrow$ amorphous carbon $\rightarrow$ tetrahedral amorphous carbon, were discussed by Ferrari \& Robertson (2000). In particular, the effects these authors observed on Raman spectroscopy as nanocrystalline graphite evolves towards amorphous carbon $(a-C)$ are:

(a) The $G$ peak decreases from 1600 to $\sim 1510 \mathrm{~cm}^{-1}$.

(b) Eq. (4) is no longer valid. The relation

$$
\frac{I(\mathrm{D})}{I(\mathrm{G})}=C^{\prime}(\lambda) \cdot L_{\mathrm{a}}^{2}
$$

where $C^{\prime}(514 \mathrm{~nm}) \approx 0.0055$, is proposed.

(c) $I(\mathrm{D}) / I(\mathrm{G})$ goes from 2 to values close to zero.

(d) Increasing dispersion of the $G$ peak occurs. The Raman spectra of hydrogenated amorphous carbon $(a-\mathrm{C}: \mathrm{H})$ will present first order peaks similar to amorphous carbon $(a-C)$, unless they are masked by photoluminescence.

Ferrari \& Robertson (2000) conclude that the G mode is related to the in-plane bond-stretching motion of pairs of $\mathrm{C} \mathrm{sp}^{2}$ atoms. This mode does not require the presence of sixfold rings. The label G (for "graphitic") is thus quite unfortunate, as this mode occurs in any $\mathrm{sp}^{2}$ paired carbon atoms. It occurs at all $\mathrm{sp}^{2}$ sites, not only those in rings, and can therefore occur in olefinic chains. The D peak is a breathing mode of $A_{1 g}$ symmetry involving phonons near the $K$ zone boundary, which becomes active in the presence of disorder, and its position depends on the photon excitation energy. Ferrari \& Robertson (2000) show that the intensity of the $\mathrm{D}$ peak is strictly connected to the presence of sixfold aromatic rings.

\subsubsection{Results from micro-Raman spectroscopy of IDPs}

The top panel of Fig. 3 shows the Raman spectra of 4 HFhydrolized IDPs: K2, N (already introduced), W7116B-Y, and 


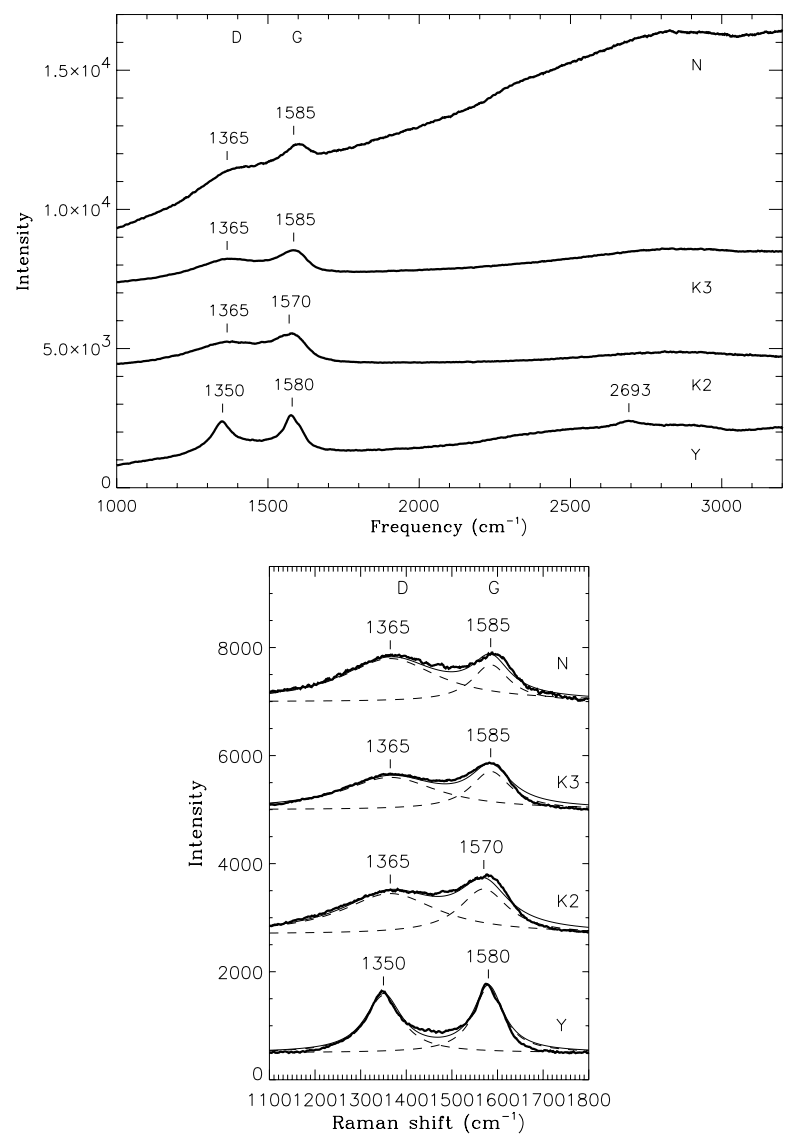

Fig. 3. Top: Raman spectra of 4 hydrolized IDPs. Bottom: blow-up of the first-order D and G bands after baseline correction (thick line) and Lorentzian fits (thin line). The intensity of the spectra was shifted for intercomparison.

L2021-K3 (abbreviated as Y and K3; see Sect. 3.3.3 for their infrared characterization). Except for IDP N, the continuum of the spectra is relatively flat, indicating that only particle $\mathrm{N}$ presents significant laser-induced fluorescence.

A blow-up of the first-order $\mathrm{D}$ and $\mathrm{G}$ bands is also given in Fig. 3 (bottom panel), after subtraction of the continuum using a spline baseline. The Lorentzian fits of the D and $G$ bands separately and the sum of both Lorentzians (providing a fit of the baseline corrected first-order spectrum) are also shown. Table 5 provides the parameter values for the fits, namely the derived $\mathrm{D}$ and $\mathrm{G}$ line positions and $F W H M$, and the intensity ratio $I(\mathrm{D}) / I(\mathrm{G})$.

In order to compare the infrared and Raman spectra of the carbonaceous fraction of IDPs, we analyzed the IDPs that were treated with HF (see Sect. 3.3.3 for explanation). The Raman parameters of these IDPs fall within the range of other IDPs studied (Wopenka 1988). The average parameter values obtained for 11 of the IDPs analyzed by Wopenka (1988), as reported by Ferini et al. (2004), are included in Table 5. For each parameter, the maximum and minimum values are given in parenthesis. These average values are similar to the ones that can be deduced from the Raman spectra of 10 other IDPs (Raynal 2003).

\subsubsection{Comparison between infrared and Raman spectra of IDPs}

The IDPs K2, N, Y and K3 were characterized in the infrared before and after HF hydrolysis (Matrajt et al. 2005). The
HF-hydrolysis of IDPs reduces their silicate contents and improves the infrared detection of functional groups in organic samples. However, it should be considered that some organics can also be altered by the HF treatment. The four IDPs contained layer-lattice silicates and have $\mathrm{CH}_{2} / \mathrm{CH}_{3}$ ratios of 3.6, 5.5, 4.2, and 2.8, respectively. The IDPs $\mathrm{N}$ and $\mathrm{K} 3$ show infrared features around $\sim 1600 \mathrm{~cm}^{-1}$ (probably due to the $\mathrm{C}=\mathrm{C}$ str. in aromatic species) and $\sim 1715 \mathrm{~cm}^{-1}$ (which could be due to the $\mathrm{C}=\mathrm{O}$ str. in ketones). The infrared spectrum of particle $\mathrm{Y}$ prior to HF hydrolysis (see Fig. 4) shows a band at $1595 \mathrm{~cm}^{-1}$ that is not observed after the hydrolysis, and no carbonyl band, while the infrared spectrum of particle K2 does not show any carbonyl or aromatic features (Matrajt et al. 2005).

The photoluminescence background is known to increase with increasing $\mathrm{H}$ content, but this is not the only cause of photoluminescence. This background overshadows the Raman signal of $a-\mathrm{C}: \mathrm{H}$ materials with $\mathrm{H}$ content over $\sim 40-45$ at.\% (Ferrari \& Robertson 2000). The significant fluorescence of IDP N, with respect to the other IDPs, might be related to the lower $\mathrm{CH}_{2} / \mathrm{CH}_{3}$ ratio (which reflects a higher degree of ramification of the aliphatic chains), giving a higher $\mathrm{H} / \mathrm{C}$ ratio, but this needs to be confirmed with a more extended statistical sample, given the very limited number of particles investigated here. It does not seem to be related to the infrared bands at $\sim 1600 \mathrm{~cm}^{-1}$ and $\sim 1715 \mathrm{~cm}^{-1}$ attributed to an aromatic component (as known, not all PAHs show fluorescence) and a carbonyl group, since these bands are also observed in particle K3, which does not fluoresce significantly.

From the comparison between infrared and Raman spectroscopy of the IDPs studied here we conclude that there is no clear correlation between the infrared band intensity around $1600 \mathrm{~cm}^{-1}$ and the intensity of the D peak in Raman. For instance, the intensity of the D peak is comparable for IDPs K2 and $\mathrm{K} 3$, while the infrared $1600 \mathrm{~cm}^{-1}$ band is only observed in K3 (Fig. 5 in Matrajt et al. 2005). The fact that the D peak is observed in the vast majority of the Raman spectra of IDPs reported in the literature proves that these particles contain a significant fraction of aromatic structures. However, given the low intrinsic strength of the aromatic bands in the infrared and the possible overlap with other bands (such as the bending mode of $\mathrm{H}_{2} \mathrm{O}$ in phyllosilicates), they are not easily detectable using micro-FTIR. On the other hand, the aliphatic component of IDPs is difficult to infer from visible Raman spectroscopy, while it is readily observed in the infrared.

\subsubsection{Characterization of the bulk of the carbonaceous IDP material}

Previous works report on the Raman spectroscopy of IDPs (Wopenka 1988; Keller et al. 1994; Raynal et al. 2000a). It was found that the first-order spectra of different IDPs are rather similar, containing broad D (around $1350 \mathrm{~cm}^{-1}$ ) and $\mathrm{G}$ (around $1580 \mathrm{~cm}^{-1}$ ) bands that are well differentiated, which indicate a poorly crystallized carbonaceous material. In this section we characterize of the bulk of the carbonaceous material in IDPs based on the recent literature on the interpretation of Raman spectra of carbon materials (Ferrari \& Robertson 2000).

From Eq. (5) in Sect. 3.3.1, the aromatic domain size, $L_{\mathrm{a}}$, corresponding to the average IDP value of $I(\mathrm{D}) / I(\mathrm{G}) \sim 1$ (Table 5), is $1.35 \mathrm{~nm}$ ( 5 to 6 rings in diameter, or a total number of $\sim 30$ rings and $\sim 80 \mathrm{C}$ atoms), an indication of a highly disordered material. For the $I(\mathrm{D}) / I(\mathrm{G})$ values of IDPs with the highest deviation, 0.66 and 1.39 (Table 5), the corresponding $L_{\mathrm{a}}$ values are 1.10 and $1.59 \mathrm{~nm}$ (i.e. a total number of 20 to 42 rings). In 
Table 5. Lorentzian fit values for the $\mathrm{D}$ and $\mathrm{G}$ bands corresponding to line position, $F W H M$, and intensity ratio $I(\mathrm{D}) / I(\mathrm{G})$.

\begin{tabular}{cccccc}
\hline \hline IDP & $\begin{array}{c}\text { D line position } \\
\left(\mathrm{cm}^{-1}\right)\end{array}$ & $\begin{array}{c}\text { G line position } \\
\left(\mathrm{cm}^{-1}\right)\end{array}$ & $\begin{array}{c}\mathrm{D} \text { line } F W H M \\
\left(\mathrm{~cm}^{-1}\right)\end{array}$ & $\begin{array}{c}\text { G line } F W H M \\
\left(\mathrm{~cm}^{-1}\right)\end{array}$ & $I(\mathrm{D}) / I(\mathrm{G})$ \\
Y & 1350 & 1580 & 96 & 80 & 0.88 \\
K2 & 1365 & 1570 & 256 & 130 & 0.90 \\
K & 1365 & 1585 & 256 & 108 & 0.84 \\
11 IDPs (average $)^{1}$ & $1362(1345-1393)$ & $1584(1562-1594)$ & $296(141-476)$ & $104(72-159)$ & $1.02(0.66-1.39)$ \\
\hline
\end{tabular}

${ }^{1}$ Average values of Raman parameters corresponding to 11 IDPs (Wopenka 1988), reported by Ferini et al. (2004).
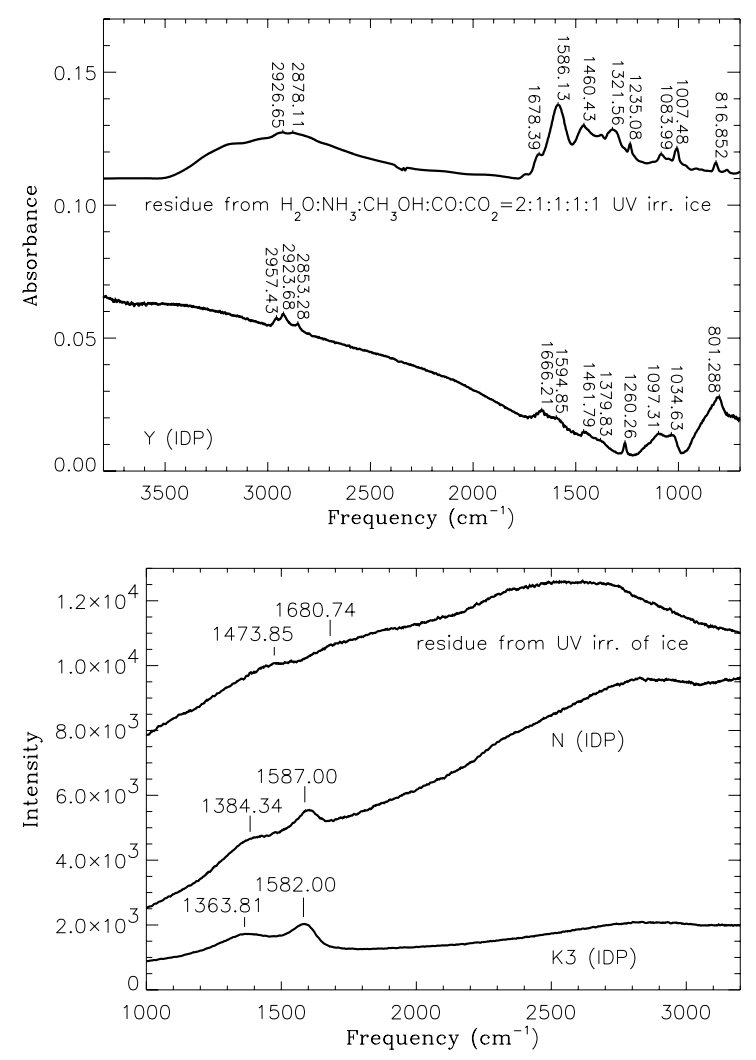

Fig. 4. Top: comparison between the infrared spectra of IDP $\mathrm{Y}$ (bottom) and a residue (top) made from UV-irradiation of the $\mathrm{H}_{2} \mathrm{O}: \mathrm{CH}_{3} \mathrm{OH}: \mathrm{CO}: \mathrm{CO}_{2}: \mathrm{NH}_{3}=2: 1: 1: 1: 1$ ice mixture (adapted from Muñoz Caro \& Schutte 2003). Bottom: comparison between the Raman spectra of IDPs K3 (with Raman parameters close to the average values of 11 IDPs, Table 5) and $\mathrm{N}$ (with a high photoluminescence) after $\mathrm{HF}$ hydrolysis, and a residue from UV-irradiation of $\mathrm{H}_{2} \mathrm{O}: \mathrm{CH}_{3} \mathrm{OH}: \mathrm{NH}_{3}=1: 1: 1$ ice.

general, the aromatic domain size of amorphous carbon should be lower than $2 \mathrm{~nm}$ (Ferrari \& Robertson 2000). From the average $\mathrm{G}$ line position in IDPs (see Table 5), $1584 \mathrm{~cm}^{-1}$, including the extreme values, 1562 and $1594 \mathrm{~cm}^{-1}$, the dispersion of this peak (average $F W H M$ value of $104 \mathrm{~cm}^{-1}$, ranging from 72 to $159 \mathrm{~cm}^{-1}$ ), and the corresponding $I(\mathrm{D}) / I(\mathrm{G})$ values (average $I(\mathrm{D}) / I(\mathrm{G})$ of 1.02 , ranging between 0.66 and 1.39$)$, it can be concluded that all the Raman spectra of IDPs reported by Wopenka (1988), Raynal (2003), and this work (excluding group 6 from Wopenka (1988) that show no first-order bands, but this might be due to the absence of carbon in the particles), indicate that the structure of the bulk of the carbonaceous material of IDPs is that of amorphous, or hydrogenated amorphous, carbon. For IDPs containing amorphous carbon $(a-\mathrm{C})$, the $\mathrm{sp}^{3}$ content varies from almost 0 to near $10 \%$ (see Fig. 7 of Ferrari \& Robertson 2000), while for IDPs containing hydrogenated amorphous carbon $(a-\mathrm{C}: \mathrm{H})$, as evidenced by the $\mathrm{C}-\mathrm{H}$ modes in the infrared, the $\mathrm{sp}^{3}$ content could be, roughly, up to about $30 \%$. The different degree of hydrogenation of the amorphous carbon in IDPs is, as we previously explained, not easy to observe by Raman spectroscopy. However, in the infrared, IDPs with a low $\mathrm{H} / \mathrm{C}$ ratio will have no, or weak, $3.4 \mu \mathrm{m}$ absorption feature, while IDPs with a high $\mathrm{H} / \mathrm{C}$ ratio (corresponding to $a-\mathrm{C}: \mathrm{H}$ ) will have a clearly observable band in that region. The non-detection of a $3.3 \mu \mathrm{m}$ band (aromatic C-H str.) in IDPs is consistent with aromatic units with a total number of 20 to 40 rings, as estimated above, where most of the peripheric $\mathrm{C}-\mathrm{H}$ bonds are replaced by aliphatic chains (IDPs containing $a-\mathrm{C}: \mathrm{H}$ ) or a carbon $\mathrm{sp}^{3}$-skeleton (IDPs containing $a-\mathrm{C}$ ).

\subsection{Comparison to laboratory analogs of molecular cloud organics and diffuse medium carbonaceous grains}

There is a good match between the $\sim 10 \mu \mathrm{m}$ feature of some GEMS contained in some IDPs and astronomical amorphous silicates, linking GEMS to interstellar molecular clouds (Bradley et al. 1999). The $\mathrm{D} / \mathrm{H}$ and ${ }^{15} \mathrm{~N}$ excesses found are associated to the carbonaceous component of IDPs, indicating this material was formed at the cold temperatures characteristic of molecular clouds (e.g. Keller et al. 2004). Matter in molecular clouds is present in the gas phase or in silicate/carbon grains of stellar origin covered by ice mantles. Given the low temperatures, $T \approx 10 \mathrm{~K}$, the formation of an organic refractory component will mainly be driven by the energetic processing of the ice mantles. Thus, it is a priori justified to compare the carbonaceous IDP component to residues obtained from irradiation (induced by cosmic rays and UV photons) and warm-up of molecular cloud ice analogs. Ice processing is also expected to take place in the outer parts of circumstellar regions, where volatiles are preserved. Since icy grain mantles are $\mathrm{O}$-rich, mainly due to the high $\mathrm{H}_{2} \mathrm{O}$ abundances, and species like $\mathrm{CH}_{3} \mathrm{OH}$ and $\mathrm{CO}$, such residues show high $\mathrm{O} / \mathrm{C}$ ratios; for the residue resulting from $\mathrm{UV}$ irradiation of $\mathrm{H}_{2} \mathrm{O}: \mathrm{CH}_{3} \mathrm{OH}: \mathrm{CO}: \mathrm{CO}_{2}: \mathrm{NH}_{3}=2: 1: 1: 1: 1$ ice, $\mathrm{O} / \mathrm{C} \simeq 0.4$ was found (Muñoz Caro \& Schutte 2003).

IDPs are also compared to hydrogenated amorphous carbon films $(a-\mathrm{C}: \mathrm{H})$, prepared under different conditions, as they are used as analogs of interstellar carbon grains in the diffuse medium (e.g. Duley \& Williams 1983; Mennella et al. 1999, 2001; Dartois et al. 2005, and ref. therein). The purpose was to gain certainty on the Raman characterization of the bulk of the carbon in IDPs as amorphous carbon (Sect. 3.4.3) and to compare the carbon in IDPs to the best analog of carbon grains in the diffuse ISM (Sect. 3.4.4). 


\subsubsection{Comparison to residues made by UV-photoprocessing and warm-up of molecular cloud/circumstellar ice analogs}

The residues are made by simultaneous deposition and UVirradiation at $T \approx 12 \mathrm{~K}$ of $\mathrm{H}_{2} \mathrm{O}, \mathrm{CH}_{3} \mathrm{OH}, \mathrm{CO}, \mathrm{CO}_{2}$, and $\mathrm{NH}_{3}$ ice mixtures in different proportions, simulating the physical conditions in dense clouds. After deposition/irradiation of the ice, the system is slowly warmed up to room temperature and the remaining organic residue is analyzed in situ by means of FTIR spectroscopy and ex situ by Raman spectroscopy (for more details on the experimental protocol, see Muñoz Caro \& Schutte 2003).

The top panel of Fig. 4 shows the infrared spectra of IDP $\mathrm{Y}$ and the residue made by UV-irradiation of the $\mathrm{H}_{2} \mathrm{O}: \mathrm{CH}_{3} \mathrm{OH}: \mathrm{CO}: \mathrm{CO}_{2}: \mathrm{NH}_{3}=2: 1: 1: 1: 1$ ice mixture (adapted from Muñoz Caro \& Schutte 2003). Residues made from ice photoprocessing contain a wide variety of organic molecules, including ammonium salts of carboxylic acids $\left[\left(\mathrm{R}-\mathrm{COO}^{-}\right)\left(\mathrm{NH}_{4}^{+}\right)\right]$, hexamethylenetetramine $\left[\left(\mathrm{CH}_{2}\right)_{6} \mathrm{~N}_{4}\right]$, amides $\left[\mathrm{H}_{2} \mathrm{NC}(=\mathrm{O})-\mathrm{R}\right]$, esters $\left[\mathrm{R}-\mathrm{C}(=\mathrm{O})-\mathrm{O}-\mathrm{R}^{\prime}\right]$, and species related to polyoxymethylene $\left[\left(-\mathrm{CH}_{2} \mathrm{O}-\right)_{n}\right]$. These spectra are generally dominated by $\mathrm{OH}\left(3500-2300 \mathrm{~cm}^{-1}\right), \mathrm{C}=\mathrm{O}\left(\sim 1700 \mathrm{~cm}^{-1}\right)$, and $\mathrm{COO}^{-}$ $\left(1586 \mathrm{~cm}^{-1}\right)$ str. features, due to the significant incorporation of O in the organic material (Muñoz Caro \& Schutte 2003, and ref. therein). The $3.4 \mu \mathrm{m}$ feature assigned to the aliphatic $\mathrm{CH}$ str., composed of just two shallow bands at $\sim 2925$ and $2875 \mathrm{~cm}^{-1}$, is quite peculiar. Its shape is mainly the effect of the electrophylic oxygen atom incorporated in the aliphatic structures (Muñoz Caro \& Dartois 2007). Clearly, it differs from the $3.4 \mu \mathrm{m}$ feature profile of IDPs, composed of three subfeatures shared by aliphatic species with no heteroatoms. Although some IDPs are also known to contain carbonyl $(\mathrm{C}=\mathrm{O})$ groups (Reffner et al. 1994; Flynn et al. 2003; Matrajt et al. 2005) and hydrocarbon chains in some IDPs host N (Keller et al. 2004), infrared absorption bands due to the $\mathrm{NH}$ and $\mathrm{CN}$ str. modes should be observable if their N/C and especially the $\mathrm{O} / \mathrm{C}$ elemental abundances were as high as in the refractory organic residues made from the UV irradiation of ice analogs in dense cloud and circumstellar regions, and the presence of $\mathrm{O}-\mathrm{H}, \mathrm{C}=\mathrm{O}$, or $\mathrm{COOH}$ functional groups, giving rise to strong infrared dipole absorptions, should dominate the infrared spectrum, with intensities that are much higher than the $3.4 \mu \mathrm{m}$ feature (C-H str.) observed in many IDPs. Due to the presence of hydrated silicates in many IDPs, to scattering effects, and to the choice of a baseline for correction that especially affects the broad $\mathrm{OH}$ stretch band, it is not easy to give a quantitative constraint of the $\mathrm{OH}$ absorption in these particles that is caused exclusively by their carbonaceous fraction. The absorption feature of the $\mathrm{C}=\mathrm{O}$ band in IDPs is, at most, comparable in optical depth to the absorption of the $3.4 \mu \mathrm{m}$ band, and is generally not observed prior to hydrolysis of the particles (Matrajt et al. 2005).

The visible Raman spectrum of these residues (Fig. 4, bottom panel) is dominated by a strong photoluminescence, presumably due to its organic nature with high $\mathrm{H} / \mathrm{C}$ and $\mathrm{O} / \mathrm{C}$ ratios, and the absence of first-order $\mathrm{D}$ and $\mathrm{G}$ bands. There might be two poorly defined bands at 1474 and $1681 \mathrm{~cm}^{-1}$. This organic macromolecular material is very different from the carbonaceous material found in IDPs, already characterized as a type of amorphous carbon (Sect. 3.3.1).

Atmospheric entry heating is expected to alter the composition and structure of the carbon fraction in IDPs. To study the effect of thermal annealing on an astrophysically relevant $\mathrm{O}$ and $\mathrm{N}$-rich organic material, we submitted a residue made by
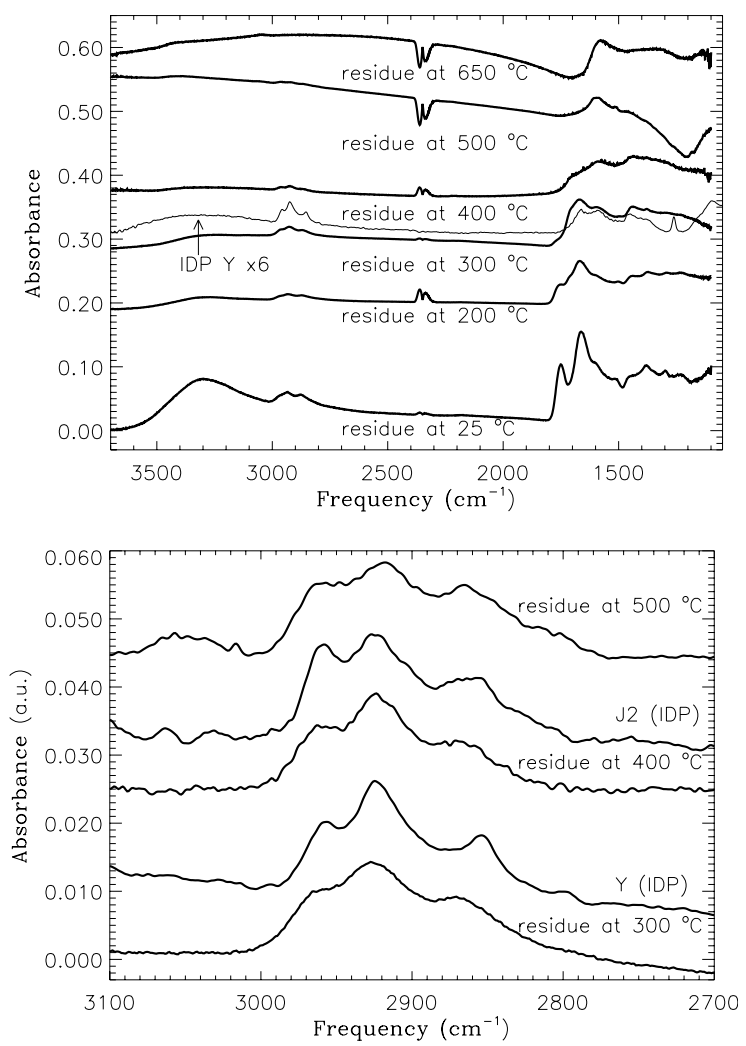

Fig. 5. Top: infrared spectra of an annealed organic residue made by UV irradiation of the $\mathrm{H}_{2} \mathrm{O}: \mathrm{CH}_{3} \mathrm{OH}: \mathrm{NH}_{3}=2: 1: 1$ ice mixture. The infrared spectrum of IDP Y is shown for comparison (thin line). Bottom: comparison between the infrared $3.4 \mu \mathrm{m}$ region of IDPs $\mathrm{Y}$ and $\mathrm{J} 2$ and residue shown in the left panel annealed at 300,400 , and $500{ }^{\circ} \mathrm{C}$. The peak optical depths are normalized for comparison and a local spline baseline was subtracted.

UV irradiation of an $\mathrm{H}_{2} \mathrm{O}: \mathrm{CH}_{3} \mathrm{OH}: \mathrm{NH}_{3}=2: 1: 1$ ice mixture to a sequence of heating steps in a vacuum oven at pressure $P \leq 1 \times 10^{-5}$ mbar. The residue was heated one hour for each temperature. After that time the oven was switched off and, once the temperature dropped to room temperature, the residue was extracted from the vacuum for micro-FTIR analysis. The results are illustrated in Fig. 5. The left panel shows the infrared spectrum of the residue at different temperatures. Carbonization due to the loss of heteroatoms, $\mathrm{O}$ and $\mathrm{N}$, is shown by the diminution of the $\mathrm{OH}$ str. band between $3600-3000 \mathrm{~cm}^{-1}$, the carbonyl $\mathrm{C}=\mathrm{O}$ str. band of esters and amides, respectively, at 1750 and $1662 \mathrm{~cm}^{-1}$, and other minor features like those at $1375(\mathrm{CH}$ scissoring) and $1225 \mathrm{~cm}^{-1}$ (CN str.). Above $300-400{ }^{\circ} \mathrm{C}$, the infrared spectrum of the residue is that of hydrogenated amorphous carbon (note the similarity with spectrum of IDP Y in the same panel, provided that the silicate band around $1000 \mathrm{~cm}^{-1}$ and the silicon oil band at $1260 \mathrm{~cm}^{-1}$ are omitted), and the $3.4 \mu \mathrm{m}$ feature between $2800-3000 \mathrm{~cm}^{-1}$ is now similar to the one observed in IDPs with $N\left(\mathrm{CH}_{2}\right) / N\left(\mathrm{CH}_{3}\right)$ ratios between 3 and 4 . The bottom panel in Fig. 5 provides a comparison between the $3.4 \mu \mathrm{m}$ feature of the residue at different temperatures, from 300 to $500{ }^{\circ} \mathrm{C}$, and that of IDPs Y and J2. This $3.4 \mu \mathrm{m}$ feature is absent above $600{ }^{\circ} \mathrm{C}$ and the weak $3.3 \mu \mathrm{m}$ feature due to the aromatic/olefinic $\mathrm{CH}$ str. becomes observable, as well as a prominent band at $1596 \mathrm{~cm}^{-1}$ due to the aromatic $\mathrm{C}=\mathrm{C}$ str. 


\subsubsection{Comparison to residues made by ion irradiation and warm-up of dense cloud and planetary system ice analogs}

Similar to the residues made by UV-irradiation of ice, discussed in Sect. 3.4.1, residues resulting from ion-irradiation of O-containing ice species (such as $\mathrm{H}_{2} \mathrm{O}$, CO, or $\mathrm{CH}_{3} \mathrm{OH}$ ) show prominent bands from the $\mathrm{O}-\mathrm{H}$ and $\mathrm{C}=\mathrm{O}$ str. modes that dominate the infrared spectrum (e.g. Strazzulla et al. 2001). Therefore, the carbonaceous fraction of IDPs differs from residues made from ion-irradiation of ice. A similar comparison was recently made by means of visible Raman spectroscopy (Ferini et al. 2004), and it found that residues resulting from ionirradiation of ice are far less ordered than IDPs. Nevertheless, as we found for the case of residues made from UV-irradiation of ice mixtures (Sect. 3.4.1), thermal annealing of residues from ion-irradiation of ice might also lead to amorphous carbon similar to IDPs.

\subsubsection{Comparison to hydrogenated carbon grains}

Hydrogenated amorphous carbon films have long been used as an analog of interstellar carbonaceous materials (Duley \& Williams 1983). We selected those with infrared and Raman spectra that were closer to the spectra of IDPs: $\mathrm{ACH} 2$ is prepared by condensation of carbon vapor formed by arc discharge between two carbon rods under a 10 mbar hydrogen atmosphere (Mennella et al. 2001), ACARL consists of carbon grains prepared by condensation of carbon vapor obtained by laser evaporation of carbon rods under a 10 mbar argon atmosphere, and ACARL_H is ACARL that is exposed to atomic hydrogen (Mennella et al. 1999).

The top panel of Fig. 6 shows a comparison between the $3.4 \mu \mathrm{m}$ feature of ACH2 and ACARL_H, and that of IDPs Y and J2. The band shape and positions are similar between IDP J2 and ACARL_H, which present comparable $N\left(\mathrm{CH}_{2}\right) / N\left(\mathrm{CH}_{3}\right)$ ratios, respectively, $2.9 \pm 0.4$ and $2.6 \pm 0.4$. The same holds for the comparison between IDP Y and ACH2. The other features observed in the infrared spectrum of hydrogenated carbon grains correspond, in the case of ACARL_H, to the $\mathrm{C}=\mathrm{C}$ skeletal vibrations of the $\mathrm{sp}^{2}$ aromatic clusters at $1580 \mathrm{~cm}^{-1}$, the $\mathrm{CH}_{2}$ and $\mathrm{CH}_{3}$ deformation modes at 1378 and $1457 \mathrm{~cm}^{-1}$, a broad band at $780 \mathrm{~cm}^{-1}$ that might be due to the phonon density of states in amorphous carbon, and the $\mathrm{C}=\mathrm{O}$ str. feature that appears $\approx 1700 \mathrm{~cm}^{-1}$ by oxydation due to air exposure (Mennella et al. 1999). The bottom panel of Fig. 6 compares IDP Y to ACARL_H in the region where the $\mathrm{C}=\mathrm{C}$ str. and the $\mathrm{CH}$ deformation bands appear. For ACARL_H, the integrated intensities of the deformation bands at 1457 and $1378 \mathrm{~cm}^{-1}$ is 14 and 35 times weaker than the whole $3.4 \mu \mathrm{m}$ feature (Mennella et al. 1999). Similar deformation mode features are observed in the spectrum of IDP Y (its unusually large organic-to-silicate ratio allows the detection of these bands), as well as a band around $1593 \mathrm{~cm}^{-1}$, which is probably due to the $\mathrm{C}=\mathrm{C}$ str. in aromatics. It is not possible to assess the presence of a band at $780 \mathrm{~cm}^{-1}$ in IDP Y, because silicates dominate that part of the spectrum, while the $\mathrm{C}=\mathrm{O}$ str. band around $\approx 1700 \mathrm{~cm}^{-1}$ caused by air contamination, is not present in the spectrum of IDP Y. We can conclude that the infrared absorbing carbonaceous component of IDP Y presents a chemical structure that resembles that of hydrogenated carbon grains. Except for the fact that the $N\left(\mathrm{CH}_{2}\right) / N\left(\mathrm{CH}_{3}\right)$ ratio can vary significantly between IDPs, most of them show bands in the $3.4 \mu \mathrm{m}$ region similar to those of IDP Y, with subfeatures of equivalent widths and positions and
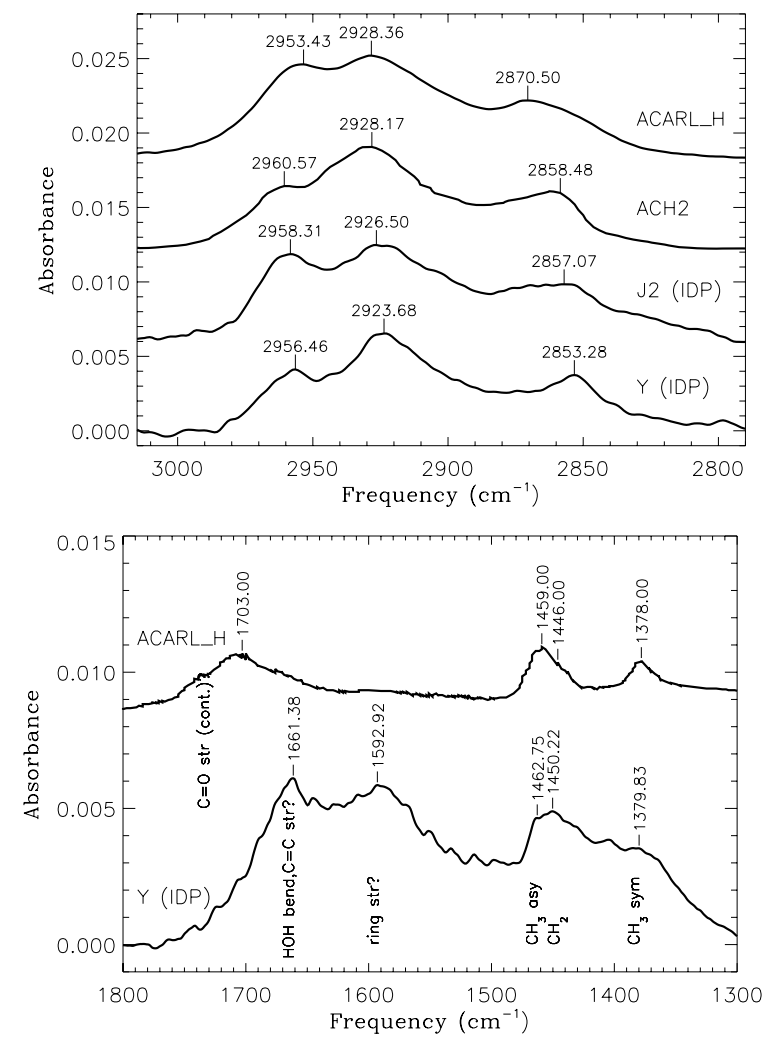

Fig. 6. Top: comparison between the infrared $3.4 \mu \mathrm{m}$ region of IDPs Y and J2, an ACH2 sample (after Mennella et al. 2001), and an ACARL_H sample (after Mennella et al. 1999). Bottom: comparison of the $\mathrm{CH}$ bending modes between IDP Y and ACARL_H (after Mennella et al. 1999). The peak optical depths are normalized for intercomparison.

no strong absorption bands from the incorporation of $\mathrm{H}$ and $\mathrm{O}$ in the carbon skeleton. It is therefore apparent that the majority of the IDPs with a substantial fraction of aliphatic carbon, relative to the silicate fraction, contain a carbonaceous component with a bulk composition that resembles hydrogenated carbon grains like ACARL_H or ACH2.

This conclusion agrees with the similarity observed between the Raman spectra of hydrogenated carbon grains and IDPs (Ferini et al. 2004). In particular, the Raman parameters of ACARL_H (see Table 6) are practically the same as for the average of the 11 IDPs given in Table 5. It can be stated that the dominant carbon structure of IDPs is analogous to ACARL (for particles with no observable infrared $3.4 \mu \mathrm{m}$ band) or to ACARL_H (for particles showing an infrared $3.4 \mu \mathrm{m}$ band). Indeed, the detailed characterization of the dominant carbon in IDPs (Sect. 3.3.4) corresponds to amorphous carbon with different degrees of hydrogenation, which is also the composition of ACARL and ACARL_H.

\subsubsection{Comparison to residues made by photoprocessing and warm-up of aliphatic species, as analogs of the $3.4 \mu \mathrm{m}$ carrier in the diffuse ISM}

UV-irradiation of ice made of simple aliphatic species with no oxygen, such as $\mathrm{CH}_{4}$, performed under interstellar conditions ( $10 \mathrm{~K}$ and $\mathrm{Ly}-\alpha$ photons) leads to the formation of a hydrogenated amorphous carbon polymer called photoproduced $(a-\mathrm{C}: \mathrm{H})$. The composition of this material must be very similar to that of carbonaceous grains in the diffuse medium, as it provides a good match of all the observed mid-infrared 
Table 6. Lorentzian fit values for the $\mathrm{D}$ and $\mathrm{G}$ bands corresponding to line position, $F W H M$, and intensity ratio $I(\mathrm{D}) / I(\mathrm{G})$ of $\mathrm{ACH} 2$, ACARL, and ACARL_H samples (adapted from Mennella et al. 2003).

\begin{tabular}{cccccc}
\hline \hline Sample & $\begin{array}{c}\text { D line position } \\
\left(\mathrm{cm}^{-1}\right)\end{array}$ & $\begin{array}{c}\text { G line position } \\
\left(\mathrm{cm}^{-1}\right)\end{array}$ & $\begin{array}{c}\text { D line } F W H M \\
\left(\mathrm{~cm}^{-1}\right)\end{array}$ & $\begin{array}{c}\mathrm{G} \text { line } F W H M \\
\left(\mathrm{~cm}^{-1}\right)\end{array}$ & $I(\mathrm{D}) / I(\mathrm{G})$ \\
\hline ACH2 & $1347 \pm 4$ & $1570 \pm 2$ & $295 \pm 11$ & $199 \pm 5$ & $0.61 \pm 0.04$ \\
ACARL & $1372 \pm 2$ & $1589 \pm 1$ & $355 \pm 7$ & $86 \pm 2$ & $1.04 \pm 0.04$ \\
ACARL_H & $1360 \pm 2$ & $1585 \pm 1$ & $284 \pm 7$ & $103 \pm 3$ & $0.96 \pm 0.06$ \\
\hline
\end{tabular}
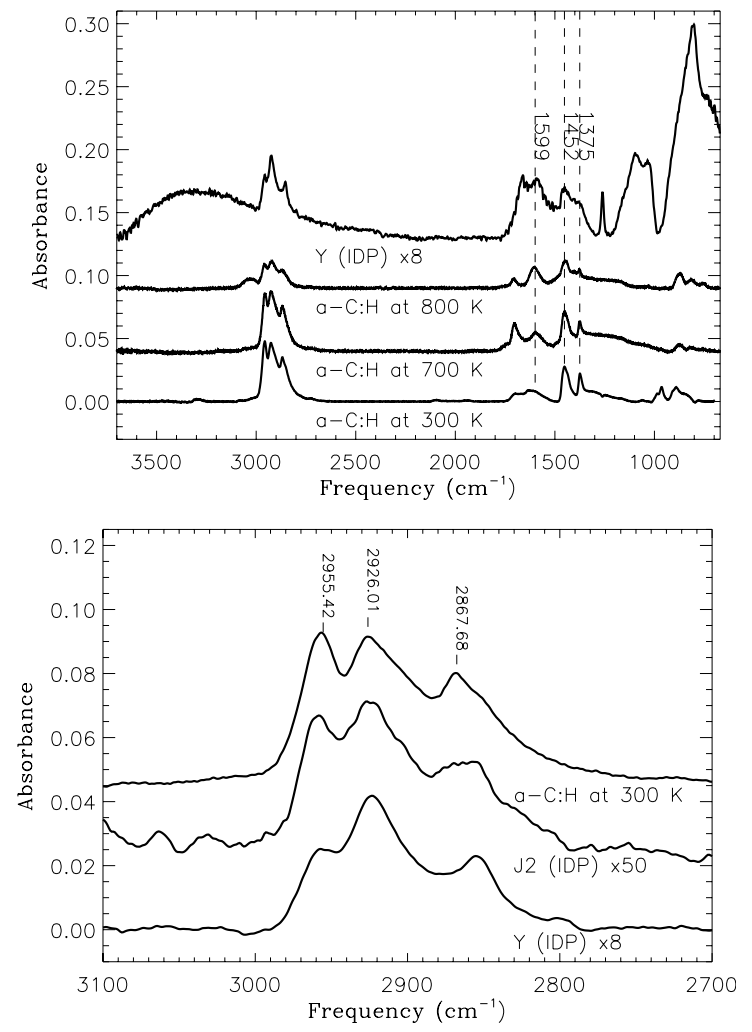

Fig. 7. Top: comparison between the infrared spectra of $a-\mathrm{C}: \mathrm{H}$ at 300 , 700, and $800 \mathrm{~K}$ (adapted from Dartois et al. 2005), and IDP Y. Bottom: comparison between the $3.4 \mu \mathrm{m}$ region of IDPs $\mathrm{Y}$ and $\mathrm{J} 2$, and $a-\mathrm{C}: \mathrm{H}$ at $300 \mathrm{~K}$.

absorption features and satisfies the ISM vapor pressure requirements, contrary to pure branched aliphatics (Dartois et al. 2004, 2005). In particular, the $3.4 \mu \mathrm{m}$ band of both materials is basically identical, and therefore differs from that observed in IDPs, as earlier found (e.g. Flynn et al. 2003, Matrajt et al. 2005). In fact, this difference is due to the different $N\left(\mathrm{CH}_{2}\right) / N\left(\mathrm{CH}_{3}\right)$ ratio. The top panel of Fig. 7 compares the infrared spectra of IDP Y to photoproduced $a-\mathrm{C}: \mathrm{H}$ at $300 \mathrm{~K}$ and annealed at $700 \mathrm{~K}$ and $800 \mathrm{~K}$. The $\mathrm{CH}$ deformation modes at 1375 and $1452 \mathrm{~cm}^{-1}$ in aliphatics, as well as a band around $1600 \mathrm{~cm}^{-1}$ that is probably due to the $\mathrm{C}=\mathrm{C}$ str. in aromatics, are observed both in IDP Y and photoproduced $a-\mathrm{C}: \mathrm{H}$. The bottom panel of Fig. 7 shows a blowup of the $3.4 \mu \mathrm{m}$ region after local subtraction of a spline baseline comparing IDPs Y and J2 to photoproduced $a-\mathrm{C}: \mathrm{H}$ at $300 \mathrm{~K}$. Although the $3.4 \mu \mathrm{m}$ feature of IDP J2 is not very well-resolved, it is close to the same feature in photoproduced $a-\mathrm{C}: \mathrm{H}$, given the relatively low $N\left(\mathrm{CH}_{2}\right) / N\left(\mathrm{CH}_{3}\right)$ ratio of 2.9 for this IDP, although still higher than the diffuse ISM value around 2.2 (Matrajt et al. 2005 and ref. therein). The Raman spectrum of photoproduced $a$-C:H (Dartois et al. 2005) corresponds to a considerably more disordered carbon structure than that of IDPs.

An interesting question is whether carbon in IDPs could originate from the diffuse medium and present higher
$N\left(\mathrm{CH}_{2}\right) / N\left(\mathrm{CH}_{3}\right)$ ratios and higher values of the aromatic domain size, due to heating during atmospheric entry. Annealing of photoproduced $a$-C:H samples that resemble diffuse interstellar carbon grains to $700 \mathrm{~K}$ does not alter the $3.4 \mu \mathrm{m}$ band shape. Further annealing to $800 \mathrm{~K}$ starts modifying this band, but a $3.3 \mu \mathrm{m}$ band due to the aromatic $\mathrm{CH}$ str. appears in the spectrum, which is not observed in most IDPs (see Fig. 7, top panel). Therefore, as suggested by Matrajt et al. (2005), atmospheric entry heating does not seem to be responsible for the higher values of the $N\left(\mathrm{CH}_{2}\right) / N\left(\mathrm{CH}_{3}\right)$ ratio observed in most IDP spectra when compared to the diffuse medium spectrum.

\section{Discussion}

This is the first study of the response of IDP matter upon exposure to UV and ion irradiation. Since IDPs of interstellar origin would have suffered intense irradiation by photons and cosmic rays, especially in the diffuse ISM and later in the interplanetary medium, the effects of such irradiation on these particles is discussed in Sect. 4.1.

A possible scenario for the origin and evolution of IDPs based on the literature and our results on the irradiation and characterization of the dominant carbon in IDPs is sketched in Sect. 4.2.

\subsection{UV and ion irradiation of IDPs in space}

The mass distribution of IDPs of interstellar origin has a maximum around $10^{-16} \mathrm{~kg}$ corresponding to radius $a \sim 0.2 \mu \mathrm{m}$ using a density $\rho=2.5 \times 10^{3} \mathrm{~kg} \mathrm{~m}^{-3}$ (Landgraf et al. 2000). Thus, interstellar IDPs are similar in size to "classical" grains in the diffuse medium $(a \geq 0.1 \mu \mathrm{m})$ and considerably smaller than the micron-sized stratospheric IDPs considered here. Particle size affects the absorption of radiation and needs to be considered when extrapolating our results on the irradiation of stratospheric IDPs to grains in the diffuse ISM.

The $3.4 \mu \mathrm{m}$ band assigned to the $\mathrm{CH}$ str. of the aliphatic component in IDPs was efficiently destroyed by hard UV irradiation. Particle J2 was exposed to a UV fluence of $3.2 \times 10^{19} \mathrm{UV}$ photons $\mathrm{cm}^{-2}$, corresponding to a residence time in the diffuse medium of $\approx 1.3 \times 10^{4} \mathrm{yr}$ (for a UV flux $\Phi_{\mathrm{UV}}=8 \times 10^{7}$ photons $\mathrm{cm}^{-2} \mathrm{~s}^{-1}$, Mathis et al. 1983), i.e. 3 orders of magnitude lower than the canonical residence time that grains spend in the diffuse medium before entering a dense cloud. Given the short range of hard UV photons for carbon materials, the aliphatic component of interstellar IDPs would also become dehydrogenated in the diffuse ISM. It was reported here that $1 \mathrm{MeV}$ protons interact with the carbon fraction of stratospheric IDPs destroying the $\mathrm{C}-\mathrm{H}$ bonds. High-energy ions with penetration ranges larger than interstellar grain sizes will also lead to dehydrogenation of the aliphatic component (e.g., from the TRIM simulation, a proton with $E \geq 11 \mathrm{keV}$ can pass through a hydrocarbon grain of $a \sim 0.1 \mu \mathrm{m}$ and elemental composition $\mathrm{C}: \mathrm{H}=1: 1$, leading to dehydrogenation). However, the diffuse 
medium flux of keV protons is not well known. The estimated flux of $\mathrm{MeV}$ protons is quite low, $\Phi_{1 \mathrm{MeV}} \approx 2 \times 10^{-3}$ protons $\mathrm{cm}^{-2} \mathrm{~s}^{-1} \mathrm{sr}^{-1}$ (Shen et al. 2004). Thus, even though the destruction cross section for $\mathrm{C}-\mathrm{H}$ bonds by $1 \mathrm{MeV}$ protons $\left(9.4 \pm 0.3 \times 10^{-16} \mathrm{~cm}^{2}(1 \mathrm{MeV} \text { proton })^{-1}\right.$ for ACARL_H $)$ is 4 orders of magnitude higher than for UV photons $(1 \times$

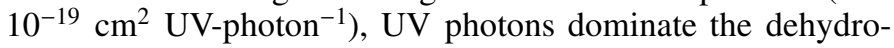
genation (Mennella et al. 2003 and ref. therein) in the diffuse medium. On the other hand, protons with $E<11 \mathrm{keV}$ will very likely lead to $\mathrm{H}$ implantation of interstellar carbon grains with $a \geq 0.1 \mu \mathrm{m}$, in a similar way to the $\mathrm{H}$ implantation observed for $20 \mathrm{keV}$ proton irradiation of a stratospheric IDP (Sect. 3.2). Also $\mathrm{H}$ atoms are expected to hydrogenate the carbonaceous component of grains in the diffuse ISM, since ACARL, an ana$\log$ material, is efficiently hydrogenated by exposure to atomic $\mathrm{H}$ (Mennella et al. 1999). Although the flux of protons with $E<11 \mathrm{keV}$ is not known, the neutral hydrogen flux alone, $\Phi_{\mathrm{H}}=8 \times 10^{6} \mathrm{H}$ atoms cm $\mathrm{cm}^{-2} \mathrm{~s}^{-1}$ (Sorrell 1990), can counteract the photon-driven dehydrogenation (Muñoz Caro et al. 2001; Mennella et al. 2001).

The conclusion is that, based on irradiation processing, interstellar IDPs from the diffuse medium entering the Solar System are expected to be hydrogenated, showing a $3.4 \mu \mathrm{m}$ feature similar to what is observed toward the Galactic Center. The fact that stratospheric IDPs present both different degrees of hydrogenation and different $\mathrm{CH}_{2} / \mathrm{CH}_{3}$ ratios, compared to diffuse medium carbon grains, and larger aromatic domain sizes (average of about 30 rings in total, see Sect. 3.3.4, compared to the expected 1-2 rings for the carrier of the $3.4 \mu \mathrm{m}$ band toward the diffuse medium, see Dartois et al. 2005) indicates that the carbon in IDPs did not originate in the diffuse ISM or that reprocessing at a later stage was very significant.

It is believed that IDPs stay $10^{4}-10^{5} \mathrm{yr}$. in the interplanetary medium, where they are photoprocessed by solar-UV photons (Strazzulla et al. 2001), leading to dehydrogenation of the IDP carbonaceous fraction. According to Strazzulla et al. (2001), IDPs are exposed to $100 \mathrm{keV}$ solar protons up to a total dose on the order of $10-100 \mathrm{eV} / \mathrm{mol}$. Also, neutral $\mathrm{H}$ is a solar wind component. Therefore, hydrogenation by $\mathrm{H}$ and $\mathrm{H}^{+}$, with energies on the order of several $\mathrm{keV}$, proceeds in the solar system, as well as dehydrogenation by the solar-UV. However, unlike in the diffuse ISM, these two processes are not necessarily in dynamical equilibrium, which could account for the different degree of hydrogenation between IDPs. Also, coagulation of IDPs into larger bodies shielded the carbonaceous material from photoprocessing, inhibiting dehydrogenation.

\subsection{Conclusions: A possible scenario for the birth and evolution of IDPs in space}

The structure and composition of the carbonaceous phases in IDPs is related to the formation and evolution of this material in space, followed by alteration during atmospheric entry heating. It is observed that the degree of order of the carbonaceous fraction for different IDPs does not differ greatly, which might be proof of a common origin and/or similar evolution of the carbon in these particles. On the other hand, the aliphatic $\mathrm{H} / \mathrm{C}$ ratio is quite diverse among IDPs, which might be due to a variable equilibrium between hydrogenation and dehydrogenation processes in space or to different degrees of annealing experienced during atmospheric entry. It was found that the chemical structure of the bulk of the carbonaceous IDP component is that of amorphous carbon (a-C), or hydrogenated amorphous carbon (a-C:H) for particles with a significant $3.4 \mu \mathrm{m}$ infrared feature.
This corresponds to a poorly graphitized carbon with an aromatic domain size between 1.1-1.6 $\mathrm{nm}$ (a total number of $\sim 20$ to 40 rings, depending on the particle), linked either by aliphatic chains with $\mathrm{N}\left(\mathrm{CH}_{2}\right) / \mathrm{N}\left(\mathrm{CH}_{3}\right)$ ratios varying from 2.8 to 5.5 for IDPs containing a-C:H (Matrajt et al. 2005, this paper), or a carbon $s p^{3}$-skeleton for IDPs containing a-C. This material presents low $\mathrm{O} / \mathrm{C}$ and $\mathrm{N} / \mathrm{C}$ ratios with respect to organic residues made from ice irradiation, although thermal annealing of the residues made from $U V$-irradiation of interstellar/circumstellar ice analogs leads to a similar form of amorphous carbon. It was shown that the observed infrared $3.4 \mu \mathrm{m}$ feature is not related to organic species containing $O$ and $N$. Instead, its shape is characteristic of hydrogenated amorphous carbon ( $a-C: H)$, in agreement with the Raman characterization.

Regarding the nature of the parent body of IDPs, whether asteroidal or cometary, the carbonaceous IDP material seems to contain a low fraction of heteroatoms (oxygen and nitrogen in particular) compared to cometary grains. The analysis of ejected dust during flyby missions to Comet P/Halley (Fomenkova et al. 1994; Fomenkova 1999) shows that a population of grains, about $50 \%$ of the total, contained $\mathrm{O} / \mathrm{C} \geq 0.5$. Recently, Kissel et al. 2004 reported low $\mathrm{O}$ and $\mathrm{H}$ contents and high $\mathrm{N}$ abundances for Comet $81 \mathrm{P} /$ Wild 2. Despite the variability of the elemental abundances in organic cometary grains, most IDPs cannot match the high $\mathrm{O}$ abundance reported for Comet $\mathrm{P} / \mathrm{Halley}$ dust, and infrared bands from functional groups containing $\mathrm{N}$ are generally not observed. This could indicate that IDPs are generally less pristine than cometary grains, as the latter appear to be more like the organic residues made from ice irradiation, with high $\mathrm{H} / \mathrm{C}, \mathrm{O} / \mathrm{C}$, and $\mathrm{N} / \mathrm{C}$ ratios. It might be thought that most IDPs have an asteroidal origin, in agreement with studies concerning the dynamical structure of the zodiacal cloud (Dermott et al. 1999). However, relative to the Murchison and Orgueil carbonaceous chondrites, IDPs show higher $\mathrm{CH}_{2} / \mathrm{CH}_{3}$ ratios (Matrajt et al. 2005). Thermal annealing of these particles after leaving the parent body, especially during atmospheric entry, could account for the smaller amount of heteroatoms compared to cometary dust, as we mimicked experimentally using organic residues made from ice photoprocessing (Sect. 3.4.1). This supports earlier ideas suggesting that vesicular carbons in IDPs probably result from thermal devolatilization of organic materials (Rietmeijer 1998).

Finally, a possible scenario for the formation and evolution of the dominant carbon in IDPs is proposed. We discuss three possible scenarios: the atmospheres of AGB stars, dense molecular clouds, and the outer parts of circumstellar disks.

The first possibility considers that the carbonaceous material in IDPs originated from carbon grains in the atmospheres of AGB stars being injected into the ISM by stellar winds. However, in this scenario, it is difficult to conceive how silicate grains could accrete carbon mantles. Silicate and carbon grains in these regions correspond to different populations. Additionally, the carbon grains observed toward the diffuse ISM probably formed in the ejecta of stars (Dartois et al. 2005), and it differs from the bulk of carbon in IDPs (Sect. 3.4.4).

The high $\mathrm{D} / \mathrm{H}$ and ${ }^{15} \mathrm{~N} /{ }^{14} \mathrm{~N}$ ratios, associated to hydrocarbons in the carbonaceous matrix of some IDPs, led some authors to conclude that these particles were formed in an interstellar molecular cloud environment (Keller et al. 2004, and references therein). There is, however, a problem with the dense molecular cloud origin of the carbon fraction of IDPs. The $3.4 \mu \mathrm{m}$ feature was not detected toward the line of sight of dense clouds. Grains in dense clouds are irradiated by UV photons and cosmic rays (Shen et al. 2004, and references therein) leading 
to dehydrogenation of organic species. In contrast to the diffuse medium, rehydrogenation is inhibited due to the reduced amount of atomic hydrogen available and the presence of icy mantles covering the grains, which prevent the replacement of the $\mathrm{CH}$ bonds, while their destruction by UV photons and cosmic rays still proceeds (Muñoz Caro et al. 2001; Mennella et al. 2001). The resulting carbon skeleton has lost the $H$ and D. This makes it less probable that the carbon fraction, observed in some cluster IDPs with a high $\mathrm{D} / \mathrm{H}$ content, originated in dense clouds.

The $\mathrm{D} / \mathrm{H}$ and ${ }^{15} \mathrm{~N} /{ }^{14} \mathrm{~N}$ enrichments, indicative of chemical processes at very cold temperatures, should not be exclusively associated to quiescent regions in dense clouds. The outer regions of protoplanetary disks also contain ices like $\mathrm{CO}$ (Thi et al. 2002), $\mathrm{H}_{2} \mathrm{O}, \mathrm{CO}_{2}$, and possibly $\mathrm{NH}_{4}^{+}$(Pontoppidan et al. 2005). The $\mathrm{DCO}^{+} / \mathrm{HCO}^{+}$and $\mathrm{H}_{2} \mathrm{D}^{+} / \mathrm{H}_{3}^{+}$ratios show a degree of deuteration comparable to dense cloud values (van Dishoeck et al. 2003).

Icy grain mantles were presumably exposed to UV irradiation at various stages of protostellar evolution, in particular during the T Tauri phase of the Sun. UV irradiation from the central star and interstellar UV led to a UV field orders of magnitude higher than the one present in dense clouds, allowing the irradiation of circumstellar ices and the formation of a refractory organic material around silicate and carbon grain cores (Muñoz Caro \& Schutte 2003 and ref. therein).

However, grain coagulation would progressively lead to larger particles, producing a stock of refractory organic material that is partially shielded from UV irradiation. Turbulent mixing of the grain material would bring it closer to the central star and heat progressively. In the T Tauri phase, the temperature is expected to be around $300 \mathrm{~K}$ at $2 \mathrm{AU}$ and around $500 \mathrm{~K}$ at $1 \mathrm{AU}$ from the central star (Markwick et al. 2002). Grain heating leads to the sublimation of the icy volatiles, while the organic material (rich in $\mathrm{H}, \mathrm{O}$ and $\mathrm{N}$ ) becomes a form of amorphous carbon with a smaller amount of heteroatoms, as we observed for residues made from ice photoprocessing (Sect. 3.4.1).

In this scenario, coagulation of the grains prevents total dehydrogenation of the original organic material; therefore, a fraction of the original aliphatic C-D (carbon-deuterium bonds) and bonds containing ${ }^{15} \mathrm{~N}$ are still preserved. The end result are particles composed of multiple silicate and carbon grains held together by a carbonaceous material, mainly amorphous carbon with only a small fraction of the original organic macromolecular structure, similar to IDPs. As coagulation increases, these particles could become parts of larger bodies.

Finally, atmospheric entry heating could affect the structure and composition of IDPs, contributing to the loss of the remaining $\mathrm{H}, \mathrm{N}$, and $\mathrm{O}$ associated to the carbon fraction. Temperatures of 300-400 ${ }^{\circ} \mathrm{C}$ are required to convert an organic residue from ice photoprocessing into the amorphous carbon with a small amount of heteroatoms found in IDPs. We thus need to study IDPs that were collected prior to atmospheric entry.

We have shown that amorphous carbon is the predominant form of carbon in the IDPs studied so far, while organic matter is only present at the percent level. At least a fraction of the comets, like Halley, are rich in CHON particles. Therefore, organic species of potential astrobiological interest seem to be more abundant in some of the observed comets than in the collected IDPs. This has implications for the role played by these objects on the delivery of prebiotic species to the early Earth.

Acknowledgements. We are grateful to M. E. Zolensky and his colleagues at the NASA JSC Curatorial Facility for kindly providing the particles used in this work. We thank P. Dumas for assistance with the use of the micro-FTIR spectrometer. W.-F. Thi participated on the discussions. The authors are also grateful to an anonymous referee for useful comments and discussions. G.M.M.C. was supported by a Marie Curie Individual Fellowship from the European Union and a Ramón y Cajal research contract from the MCYT.

\section{References}

Bajt, S., Chapman, H. N., Flynn, G. J., \& Keller, L. P. 1996, Lunar Plan. Sci., 27, 57

Bernas, H., et al. 1992, Nucl. Inst. Meth., B62, 416

Bradley, J. P., Humecki, H. J., \& Germani, M. S. 1992, ApJ, 394, 643

Bradley, J. P., Keller, L. P., Snow, T. P., et al. 1999, Science, 285, 1716

Brownlee, D. E. 1978, in Cosmic Dust, ed. J. A. M. McDonnell (N.Y.: J. Wiley), 295

Brucato, J. R., Strazzulla, G., Baratta, G., \& Colangeli, L. 2004, A\&A, 413, 395

Chauvin, N., et al. 2004, Nucl. Inst. Meth. A, 521, 149

Day, K. L. 1977, MNRAS, 178, 49

Dartois, E., Muñoz Caro, G. M., Deboffle, D., \& d'Hendecourt, L. 2004, A\&A, 423, L33

Dartois, E., Muñoz Caro, G. M., Deboffle, D., Montagnac, G., \& d'Hendecourt, L. 2005, A\&A, 423, L33

Demyk, K., d'Hendecourt, L., Leroux, H., Jones, A. P., \& Borg, J. 2004, A\&A, 420, 233

Dermott, S. F., Grogan, K., Holmes, E., \& Kortenkamp, S. 1999, in Formation and Evolution of Solids in Space, ed. J. Mayo Greenberg, \& Aigen Li. (Kluwer Academic Publishers), 565

Duley, W. W., \& Williams, D. A. 1983, MNRAS, 205, 67

Ferini, G., Baratta, G. A., \& Palumbo, M. E. 2004, A\&A, 414, 757

Ferrari, A. C., \& Robertson, J. 2000, Phys. Rev. B, 61, 14095

Flynn, G. J., Keller, L. P., Feser, M., Wirick, S., \& Jacobsen, C. 2003, Geochim. Cosmochim. Acta, 67, 4791

Flynn, G. J., Keller, L. P., Jacobsen, C., \& Wirick, S. 2004, Adv. Space Res., 33, 57

Fomenkova, M. N. 1999, Space Sci. Rev., 90, 109

Fomenkova, M. N., Chang, S., \& Mukhin, L. M. 1994, Geochim. Cosmochim. Acta, 58, 4503

Gotoh, Y., \& Kajiura, S. 1999, J. Nucl. Mat., 266, 1051

Keller, L. P., Thomas, K. L., \& McKay, D. S. 1994, Carbon in primitive interplanetary dust particles, in Analysis of Interplanetary Dust, ed. M. E. Zolensky, T. L. Wilson, F. J. M. Rietmeijer, \& G. J. Flynn, AIP Conf. Proc., 310, 159

Keller, L. P., Messenger, S., Flynn, G. J., et al. 2004, Geochim. Cosmochim. Acta, 68, 2577

Kissel, J., Krueger, F. R., Silén, J., \& Clark, B. C. 2004, Science, 304, 1774

Markwick, A. J., Ilgner, M., Millar, T. J., \& Henning, Th. 2002, A\&A, 385, 632

Mathis, J. S., Mezger, P., \& Panagia, N. 1983, A\&A, 128, 212

Matrajt, G., Muñoz Caro, G. M., Dartois, E., et al. 2005, A\&A, 433, 979

Mennella, V., Brucato, J. R., Colangeli, L., \& Palumbo, P. 1999, ApJ, 524, L71

Mennella, V., Muñoz Caro, G. M., Ruiterkamp, R., et al. 2001, A\&A, 367, 355

Mennella, V., Baratta, G. A., Esposito, A., Ferini, G., \& Pendleton, Y. J. 2003, ApJ, 587, 727

Muñoz Caro, G. M., Ruiterkamp, R., Schutte, W. A., Greenberg, J. M., \& Mennella, V. 2001, A\&A, 367, 347

Muñoz Caro, G. M., \& Schutte, W. A. 2003, A\&A, 412, 121

Muñoz Caro, G. M., \& Dartois, E. 2007, in prep.

Pontoppidan, K. M., Dullemond, C. P., van Dishoeck, E. F., et al. 2005, ApJ, 622,463

Raynal, P. I. 2003, Ph.D. Thesis

Raynal, P. I., Quirico, E., Borg, J., \& D’Hendecourt, L. 2000a, Lunar Plan. Sci. XXXI, abs. No. 1318

Raynal, P. I., Quirico, E., Borg, J., et al. 2000b, Planet. Space Sci., 48, 1329

Reffner, J., Carr, G. L., Sutton, S., Hemley, R. J., \& Williams, G. P. 1994, Synch. Radiation News, 7, 30

Rietmeijer, F. J. M. 1998, Planetary Materials, in Rev. Mineral., 36, ed. J. J. Papike (Washington: Mineralogical Society of America), 2-1

Sandford, S. A., \& Walker, R. M. 1985, ApJ, 291, 838

Schramm, L. S., Brownlee, D. E., \& Wheelock, M. M. 1989, Meteoritics, 24, 99

Shen, C. J., Greenberg, J. M., Schutte, W. A., \& van Dishoeck, E. F. 2004, A\&A, 415,203

Sorrell, W. H. 1990, MNRAS, 243, 570

Strazzulla, G., Baratta, G. A., \& Palumbo, M. E. 2001, Spectrochim. Acta Part A, 57,825

Thi, W. F., Pontoppidan, K. M., van Dishoeck, E. F., Dartois, E., \& d'Hendecourt, L. 2002, A\&A, 394, L27

Tuinstra, F., \& Koenig, J. L. 1970, J. Chem. Phys., 53, 23

van Dishoeck, E. F., Thi, W.-F., \& van Zadelhoff, G.-J. 2003, A\&A, 400, L1

Weber, P., \& Greenberg, J. M. 1985, Nature, 316, 403

Witt, A. N., Gordon, K. D., \& Furton, D. G. 1998, ApJ, 501, L111

Wopenka, B. 1988, Earth. Planet. Sci. Lett., 88, 221 